\title{
Sigma-model for Generalized Composite p-branes
}

\author{
V. D. Ivashchuk and V. N. Melnikov \\ Center for Gravitation and Fundamental Metrology \\ VNIIMS, 3-1 M. Ulyanovoy Str. \\ Moscow, 117313, Russia \\ e-mail: melnikov@fund.phys.msu.su
}

\begin{abstract}
A multidimensional gravitational model containing several dilatonic scalar fields and antisymmetric forms is considered. The manifold is chosen in the form $M=$ $M_{0} \times M_{1} \times \ldots \times M_{n}$, where $M_{i}$ are Einstein spaces $(i \geq 1)$. The block-diagonal metric is chosen and all fields and scale factors of the metric are functions on $M_{0}$. For the forms composite (electro-magnetic) p-brane ansatz is adopted. The model is reduced to gravitating self-interacting sigma-model with certain constraints. In pure electric and magnetic cases the number of these constraints is $n_{1}\left(n_{1}-1\right) / 2$ where $n_{1}$ is number of 1 -dimensional manifolds among $M_{i}$. In the "electro-magnetic" case for $\operatorname{dim} M_{0}=1,3$ additional $n_{1}$ constraints appear. A family of "MajumdarPapapetrou type" solutions governed by a set of harmonic functions is obtained, when all factor-spaces $M_{\nu}$ are Ricci-flat. These solutions are generalized to the case of non-Ricci-flat $M_{0}$ when also some additional "internal" Einstein spaces of non-zero curvature are added to $M$. As an example exact solutions for $D=11$ supergravity and related 12-dimensional theory are presented.
\end{abstract}

PACS number(s): 04.50.+h, 98.80.Hw, 04.60.Kz 


\section{Introduction}

At present there exists an interest in studying of so-called $p$-brane solutions in multidimensional models [8]-33] (for a review see [30]). These solutions generalize well-known Majumdar-Papapetrou solutions [34, 35] to the case when several antisymmetric forms and dilatonic scalar fields are considered.

In this paper we continue our investigation of p-brane solutions [26, 33] based on the $\sigma$-model approach to the composite electro-magnetic case, i.e. when antisymmetric forms are sums of elementary solutions. Solutions of such type are objects of intensive investigations in $D=10,11$ supergravities [1, 2] (and in theory of superstrings and $M$ -

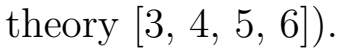

Here we obtain the $\sigma$-model representation for the composite electro-magnetic p-brane ansatz in the multidimensional gravitational model with several scalar fields and antisymmetric fields (forms). The manifold is chosen in the form $M=M_{0} \times M_{1} \times \ldots \times M_{n}$, where $M_{i}$ are Einstein spaces $(i \geq 1)$. In opposite to non-composite case 26 here a set of constraints on $\sigma$-model fields appears. These constraints occur due to non-diagonality of the stress-energy tensor $T_{N}^{M}$.

For Ricci-flat $M_{i}, i \geq 1$, we obtain ¿from the $\sigma$-model representation a set of MajumdarPapapetrou-type solutions, using the relations for scalar products of some vectors in "midisuperspace" metric. Thus, here like in [26] we extend our approach used in multidimensional cosmology (based on reduction to Toda-like systems) to $\sigma$-models of special type generated by interacting scalar, gravitational fields and generalized electro-magnetic fields (forms). For flat $M_{i}, i \geq 1$, in certain special cases our solutions agree with those considered earlier (see, for example [28, 29, 32] and references therein). The solutions are presented in the form generalizing harmonic function rule of [18] (see also [17, 19]).

Here we generalize the obtained solutions to the case of non-Ricci-flat $M_{0}$ when also some "internal" Einstein spaces of non-zero curvature are also added. In this case we "split" finding exact solutions in two parts: (i) first we solve the "background" field equations for the metric and scalar fields obeying certain "p-brane restrictions" and then (ii) we construct the generalized intersecting p-brane solutions governed by a set of harmonic functions on $M_{0}$ with the background metric.

We also note that here we start with a metric of arbitrary signature. This may have applications for supergravitational models with several times [48, 50]. Also, we consider a scalar field kinetic term of arbitrary signature (such situation takes place, for example, in 12-dimensional model from [49] that may correspond to F-theory [7]).

\section{The model}

We consider the model governed by an action

$$
\begin{aligned}
S= & \frac{1}{2 \kappa^{2}} \int_{M} d^{D} z \sqrt{|g|}\left\{R[g]-2 \Lambda-C_{\alpha \beta} g^{M N} \partial_{M} \varphi^{\alpha} \partial_{N} \varphi^{\beta}\right. \\
& \left.-\sum_{a \in \Delta} \frac{\theta_{a}}{n_{a} !} \exp \left[2 \lambda_{a}(\varphi)\right]\left(F^{a}\right)_{g}^{2}\right\}+S_{G H},
\end{aligned}
$$


where $g=g_{M N} d z^{M} \otimes d z^{N}$ is the metric $(M, N=1, \ldots, D), \varphi=\left(\varphi^{\alpha}\right) \in \mathbb{R}^{l}$ is a vector from dilatonic scalar fields, $\left(C_{\alpha \beta}\right)$ is a non-degenerate $l \times l$ matrix $(l \in \mathbb{N}), \theta_{a}= \pm 1$,

$$
F^{a}=d A^{a}
$$

is a $n_{a}$-form $\left(n_{a} \geq 2\right)$ on a $D$-dimensional manifold $M, \Lambda$ is a cosmological constant and $\lambda_{a}$ is a 1 -form on $\mathbb{R}^{l}: \lambda_{a}(\varphi)=\lambda_{a \alpha} \varphi^{\alpha}, a \in \Delta, \alpha=1, \ldots, l$. In (2.1) we denote $|g|=\left|\operatorname{det}\left(g_{M N}\right)\right|$

$$
\left(F^{a}\right)_{g}^{2}=F_{M_{1} \ldots M_{n_{a}}}^{a} F_{N_{1} \ldots N_{n_{a}}}^{a} g^{M_{1} N_{1}} \ldots g^{M_{n_{a}} N_{n_{a}}}
$$

$a \in \Delta$, where $\Delta$ is some finite set, and $S_{\mathrm{GH}}$ is the standard Gibbons-Hawking boundary term [44. In the models with one time all $\theta_{a}=1$ when the signature of the metric is $(-1,+1, \ldots,+1)$.

The equations of motion corresponding to (2.1) have the following form

$$
\begin{array}{r}
R_{M N}-\frac{1}{2} g_{M N} R=T_{M N}-\Lambda g_{M N} \\
\triangle[g] \varphi^{\alpha}-\sum_{a \in \Delta} \theta_{a} \frac{\lambda_{a}^{\alpha}}{n_{a} !} e^{2 \lambda_{a}(\varphi)}\left(F^{a}\right)_{g}^{2}=0 \\
\nabla_{M_{1}}[g]\left(e^{2 \lambda_{a}(\varphi)} F^{a, M_{1} \ldots M_{n_{a}}}\right)=0
\end{array}
$$

$a \in \Delta ; \alpha=1, \ldots, l$. In (2.5) $\lambda_{a}^{\alpha}=C^{\alpha \beta} \lambda_{\beta a}$, where $\left(C^{\alpha \beta}\right)$ is matrix inverse to $\left(C_{\alpha \beta}\right)$. In (2.4)

$$
T_{M N}=T_{M N}[\varphi, g]+\sum_{a \in \Delta} \theta_{a} e^{2 \lambda_{a}(\varphi)} T_{M N}\left[F^{a}, g\right]
$$

where

$$
\begin{array}{r}
T_{M N}[\varphi, g]=C_{\alpha \beta}\left(\partial_{M} \varphi^{\alpha} \partial_{N} \varphi^{\beta}-\frac{1}{2} g_{M N} \partial_{P} \varphi^{\alpha} \partial^{P} \varphi^{\beta}\right) \\
T_{M N}\left[F^{a}, g\right]=\frac{1}{n_{a} !}\left[-\frac{1}{2} g_{M N}\left(F^{a}\right)_{g}^{2}+n_{a} F_{M M_{2} \ldots M_{n_{a}}}^{a} F_{N}^{a, M_{2} \ldots M_{n_{a}}}\right] .
\end{array}
$$

In (2.5), (2.6) $\triangle[g]$ and $\nabla[g]$ are Laplace-Beltrami and covariant derivative operators respectively corresponding to $g$.

Let us consider the manifold

$$
M=M_{0} \times M_{1} \times \ldots \times M_{n}
$$

with the metric

$$
g=e^{2 \gamma(x)} g^{0}+\sum_{i=1}^{n} e^{2 \phi^{i}(x)} g^{i},
$$

where $g^{0}=g_{\mu \nu}^{0}(x) d x^{\mu} \otimes d x^{\nu}$ is an arbitrary metric with any signature on the manifold $M_{0}$ and $g^{i}=g_{m_{i} n_{i}}^{i}\left(y_{i}\right) d y_{i}^{m_{i}} \otimes d y_{i}^{n_{i}}$ is a metric on $M_{i}$ satisfying the equation

$$
R_{m_{i} n_{i}}\left[g^{i}\right]=\xi_{i} g_{m_{i} n_{i}}^{i}
$$


$m_{i}, n_{i}=1, \ldots, d_{i} ; \xi_{i}=$ const, $i=1, \ldots, n$. Thus, $\left(M_{i}, g^{i}\right)$ are Einstein spaces. The functions $\gamma, \phi^{i}: M_{0} \rightarrow \mathbf{R}$ are smooth. Here we denote $d_{\nu}=\operatorname{dim} M_{\nu} ; \nu=0, \ldots, n$. $D=\sum_{\nu=0}^{n} d_{\nu}$. We claim any manifold $M_{\nu}$ to be oriented and connected. Then the volume $d_{i}$-form

$$
\tau_{i} \equiv \sqrt{\left|g^{i}\left(y_{i}\right)\right|} d y_{i}^{1} \wedge \ldots \wedge d y_{i}^{d_{i}}
$$

and signature parameter

$$
\varepsilon(i) \equiv \operatorname{sign}\left(\operatorname{det}\left(g_{m_{i} n_{i}}^{i}\right)\right)= \pm 1
$$

are correctly defined for all $i=1, \ldots, n$.

Let $\Omega=\Omega(n)$ be a set of all non-empty subsets of $\{1, \ldots, n\}$. The number of elements in $\Omega$ is $|\Omega|=2^{n}-1$. For any $I=\left\{i_{1}, \ldots, i_{k}\right\} \in \Omega, i_{1}<\ldots<i_{k}$, we denote

$$
\begin{gathered}
\tau(I) \equiv \tau_{i_{1}} \wedge \ldots \wedge \tau_{i_{k}}, \\
M_{I} \equiv M_{i_{1}} \times \ldots \times M_{i_{k}}, \\
d(I) \equiv \sum_{i \in I} d_{i}=d_{i_{1}}+\ldots+d_{i_{k}},
\end{gathered}
$$

where $d_{i}$ is both, the dimension of the oriented manifold $M_{i}$ and the rank of the volume form $\tau_{i}$.

\section{Ansatz for composite electric p-branes}

Let $\Delta_{e} \subset \Delta$ be a non-empty subset, and

$$
\begin{aligned}
j_{e}: \Delta_{e} & \rightarrow P_{*}(\Omega) \\
a & \mapsto \Omega_{a, e} \in \Omega, \quad \Omega_{a, e} \neq \emptyset
\end{aligned}
$$

a map from $\Delta_{e}$ into the set $P_{*}(\Omega)$ of all non-empty subsets of $\Omega$, satisfying the condition

$$
d(I)+1=n_{a}
$$

for all $I \in \Omega_{a, e}, a \in \Delta_{e}$. In the following we fix the map (2.18).

For the potential forms $A^{a}, a \in \Delta_{e}$, we make the ansatz

$$
\begin{array}{r}
A^{a}=A^{a, e}=0, \quad a \in \Delta \backslash \Delta_{e}, \\
A^{a}=A^{a, e}=\sum_{I \in \Omega_{a, e}} A^{a, e, I}, \quad a \in \Delta_{e},
\end{array}
$$

where, with $\tau(I)$ from (2.15),

$$
A^{a, e, I}=\Phi^{a, e, I}(x) \tau(I)
$$

are elementary, electric type potential forms, with functions $\Phi^{a, e, I}$ smooth on $M_{0}, I \in \Omega_{a, e}$, $a \in \Delta_{e}$.

It follows from (2.20)-(2.22) that

$$
\begin{array}{r}
F^{a}=F^{a, e}=0, \quad a \in \Delta \backslash \Delta_{e} \\
F^{a}=F^{a, e}=\sum_{I \in \Omega_{a, e}} F^{a, e, I}, \quad a \in \Delta_{e},
\end{array}
$$


where

$$
F^{a, e, I}=d A^{a, e, I}=d \Phi^{a, e, I} \wedge \tau(I) .
$$

Due to (2.19) this relation is indeed self-consistent.

For dilatonic scalar fields we put

$$
\varphi^{\alpha}=\varphi^{\alpha}(x)
$$

$\alpha=1, \ldots, l$. Thus, in our ansatz all fields depend just on the point $x \in M_{0}$.

Remark 1. It is more correct to write in (2.11) $\hat{g}^{\alpha}$ instead $g^{\alpha}$, where $\hat{g}^{\alpha}=p_{\alpha}^{*} g^{\alpha}$ is the pullback of the metric $g^{\alpha}$ to the manifold $M$ by the canonical projection: $p_{\alpha}: M \rightarrow M_{\alpha}$, $\alpha=0, \ldots, n$. Analogously, we should write $\hat{\Phi}^{a, e, I}$ and $\hat{\tau}(I)$ instead $\Phi^{a, e, I}$ and $\tau(I)$ in (2.22) and (2.25). In what follows we omit all "hats" in order to simplify notations.

\section{Ricci-tensor components}

The nonzero Ricci tensor components for the metric (2.11) are the following 39

$$
\begin{array}{r}
R_{\mu \nu}[g]=R_{\mu \nu}\left[g^{0}\right]+g_{\mu \nu}^{0}\left[-\Delta_{0} \gamma+\left(2-d_{0}\right)(\partial \gamma)^{2}-\partial \gamma \sum_{j=1}^{n} d_{j} \partial \phi^{j}\right] \\
+\left(2-d_{0}\right)\left(\gamma_{; \mu \nu}-\gamma_{, \mu} \gamma_{, \nu}\right)-\sum_{i=1}^{n} d_{i}\left(\phi_{; \mu \nu}^{i}-\phi_{, \mu}^{i} \gamma_{, \nu}-\phi_{, \nu}^{i} \gamma_{, \mu}+\phi_{, \mu}^{i} \phi_{, \nu}^{i}\right), \\
R_{m_{i} n_{i}}[g]=R_{m_{i} n_{i}}\left[g^{i}\right]-e^{2 \phi^{i}-2 \gamma} g_{m_{i} n_{i}}^{i}\left\{\Delta_{0} \phi^{i}+\left(\partial \phi^{i}\right)\left[\left(d_{0}-2\right) \partial \gamma+\sum_{j=1}^{n} d_{j} \partial \phi^{j}\right]\right\},
\end{array}
$$

Here $\partial \beta \partial \gamma \equiv g^{0}{ }^{\mu \nu} \beta_{, \mu} \gamma_{, \nu}$ and $\Delta_{0}$ is the Laplace-Beltrami operator corresponding to $g^{0}$. The scalar curvature for (2.11) is [39]

$$
\begin{array}{r}
R[g]=\sum_{i=1}^{n} e^{-2 \phi^{i}} R\left[g^{i}\right]+e^{-2 \gamma}\left\{R\left[g^{0}\right]-\sum_{i=1}^{n} d_{i}\left(\partial \phi^{i}\right)^{2}\right. \\
\left.-\left(d_{0}-2\right)(\partial \gamma)^{2}-(\partial f)^{2}-2 \Delta_{0}(f+\gamma)\right\}
\end{array}
$$

where

$$
f=f(\gamma, \phi)=\left(d_{0}-2\right) \gamma+\sum_{j=1}^{n} d_{j} \phi^{j}
$$

\section{$\sigma$-model representation}

Restriction 1. Let us start first from the case

$$
n_{1} \equiv\left|\left\{i \mid d_{i}=1, i \geq 1\right\}\right| \leq 1 \text {, }
$$

i.e. the number of 1-dimensional manifolds $M_{i}, i>0$, in 2.10) is not more than 1. In this case the energy momentum tensor $T_{M N}$ from (2.7) has a block diagonal form (see Subsect. 4.2 below) that assures the existence of a $\sigma$-model representation. In Subsection 4.2 the restriction (2.31) will be omitted. 
Using (2.27), (2.28) and (2.31), it is not difficult to verify (see Proposition 2 and Remark 2 in Sect. 6 below) that the field equations (2.4)-(2.6) for the field configurations ¿from (2.11), (2.23)-(2.25) and (2.26) may be obtained as the equations of motion from the action

$$
\begin{aligned}
S_{\sigma}= & S_{\sigma}\left[g^{0}, \gamma, \phi, \varphi, \Phi\right]=\frac{1}{2 \kappa_{0}^{2}} \int_{M_{0}} d^{d_{0}} x \sqrt{\left|g^{0}\right|} e^{f(\gamma, \phi)}\left\{R\left[g^{0}\right]-\sum_{i=1}^{n} d_{i}\left(\partial \phi^{i}\right)^{2}\right. \\
& \left.-\left(d_{0}-2\right)(\partial \gamma)^{2}+(\partial f) \partial(f+2 \gamma)+\sum_{i=1}^{n} \xi_{i} d_{i} e^{-2 \phi^{i}+2 \gamma}-2 \Lambda e^{2 \gamma}-\mathcal{L}\right\}
\end{aligned}
$$

where

$$
\begin{array}{r}
\mathcal{L}=\mathcal{L}_{\varphi}+\mathcal{L}_{A}, \\
\mathcal{L}_{\varphi}=C_{\alpha \beta} \partial \varphi^{\alpha} \partial \varphi^{\beta}, \\
\mathcal{L}_{A}=\mathcal{L}_{A, e}=\sum_{a \in \Delta_{e}} \theta_{a} \sum_{I \in \Omega_{a, e}} \varepsilon(I) \exp \left(2 \lambda_{a}(\varphi)-2 \sum_{i \in I} d_{i} \phi^{i}\right)\left(\partial \Phi^{a, e, I}\right)^{2},
\end{array}
$$

where $\left|g^{0}\right|=\left|\operatorname{det}\left(g_{\mu \nu}^{0}\right)\right|$ and similar notations are applied to the metrics $g^{i}, i=1, \ldots, n$. In $(2.35)$

$$
\varepsilon(I) \equiv \varepsilon\left(i_{1}\right) \times \ldots \times \varepsilon\left(i_{k}\right)= \pm 1
$$

for $I=\left\{i_{1}, \ldots, i_{k}\right\} \in \Omega, i_{1}<\ldots<i_{k}($ see $(2.14)$ ).

For finite internal space volumes (e.g. compact $M_{i}$ ) $V_{i}$ the action (2.32) (with $\mathcal{L}$ from (2.33)) coincides with the action (2.1), i.e.

$$
S_{\sigma}\left[g^{0}, \gamma, \phi, \varphi, \Phi\right]=S\left[g\left(g^{0}, \gamma, \phi\right), \varphi, F(\Phi)\right],
$$

where $g=g\left(g^{0}, \gamma, \phi\right)$ and $F=F(\Phi)$ are defined by the relations (2.11) and (2.23)-(2.25) respectively and

$$
\kappa^{2}=\kappa_{0}^{2} \prod_{i=1}^{n} V_{i} .
$$

This may be readily verified using the scalar curvature decomposition

$$
\begin{aligned}
R[g]= & \sum_{i=1}^{n} e^{-2 \phi^{i}} R\left[g^{i}\right]+e^{-2 \gamma}\left\{R\left[g^{0}\right]-\sum_{i=1}^{n} d_{i}\left(\partial \phi^{i}\right)^{2}\right. \\
& \left.-\left(d_{0}-2\right)(\partial \gamma)^{2}+(\partial f) \partial(f+2 \gamma)+R_{B}\right\},
\end{aligned}
$$

where

$$
R_{B}=\left(1 / \sqrt{\left|g^{0}\right|}\right) e^{-f} \partial_{\mu}\left[-2 e^{f} \sqrt{\left|g^{0}\right|} g^{0} \mu \nu \partial_{\nu}(f+\gamma)\right]
$$

gives rise to the Gibbons-Hawking boundary term

$$
S_{\mathrm{GH}}=\frac{1}{2 \kappa^{2}} \int_{M} d^{D} z \sqrt{|g|}\left\{-e^{-2 \gamma} R_{B}\right\} .
$$

We note that (2.35) appears due to the relation

$$
\frac{1}{n_{a} !}\left(F^{a, e, I}\right)^{2}=\varepsilon(I) \exp \left(-2 \gamma-2 \sum_{i \in I} d_{i} \phi^{i}\right)\left(\partial \Phi^{a, e, I}\right)^{2},
$$

$I \in \Omega_{a, e}, a \in \Delta_{e}$. 


\section{Exact solutions}

First we consider the case $d_{0} \neq 2$. In order to simplify the action (2.32), we use, as in ref. [26, 39], for $d_{0} \neq 2$ the generalized harmonic gauge

$$
\gamma=\gamma_{0}(\phi)=\frac{1}{2-d_{0}} \sum_{i=1}^{n} d_{i} \phi^{i}
$$

It means that $f=f\left(\gamma_{0}, \phi\right)=0$. This gauge does not exist for $d_{0}=2$. For the cosmological case with $d_{0}=1$ and $g^{0}=-d t \otimes d t$, the gauge (3.1) is the harmonic-time gauge [41, 42] (for spherical symmetry see K.A.Bronnikov, 1973 [35]).

¿From equations (2.32), (2.35), (3.1) we get

$$
\begin{array}{r}
S_{0}\left[g^{0}, \phi, \varphi, \Phi\right]=S_{\sigma}\left[g^{0}, \gamma_{0}(\phi), \phi, \varphi, \Phi\right]=\frac{1}{2 \kappa_{0}^{2}} \int_{M_{0}} d^{d_{0}} x \sqrt{\left|g^{0}\right|}\left\{R\left[g^{0}\right]\right. \\
\left.-G_{i j} g^{0}{ }^{\mu \nu} \partial_{\mu} \phi^{i} \partial_{\nu} \phi^{j}-2 V(\phi)-\mathcal{L}\right\}
\end{array}
$$

where

$$
G_{i j}=d_{i} \delta_{i j}+\frac{d_{i} d_{j}}{d_{0}-2}
$$

are the components of the ("purely gravitational") midisuperspace metric on $\mathbf{R}^{n}$ [39] (or the gravitational part of target space metric), $i, j=1, \ldots, n$, and

$$
V=V(\phi)=\Lambda e^{2 \gamma_{0}(\phi)}-\frac{1}{2} \sum_{i=1}^{n} \xi_{i} d_{i} e^{-2 \phi^{i}+2 \gamma_{0}(\phi)}
$$

is the potential and $\mathcal{L}$ defined in (2.33). This is the action of a self-gravitating $\sigma$ model on $M_{0}$ with a $\left(n+l+\sum_{a \in \Delta_{e}}\left|\Omega_{a, e}\right|\right)$-dimensional target space and a self-interaction described by the potential (3.4).

\section{$3.1 \sigma$-model with zero potential.}

Now we consider the case $\xi_{i}=\Lambda=0$, i.e. all spaces $\left(M_{i}, g^{i}\right)$ are Ricci-flat, $i=1, \ldots, n$, and the cosmological constant is zero. In this case the potential (3.4) is trivial and we are led to the $\sigma$-model with the action

$$
S_{\sigma}=\int_{M_{0}} d^{d_{0}} x \sqrt{\left|g^{0}\right|}\left\{R\left[g^{0}\right]-\hat{G}_{A B} \partial \sigma^{A} \partial \sigma^{B}-\sum_{s \in S} \varepsilon_{s} e^{2 L_{A s} \sigma^{A}}\left(\partial \Phi^{s}\right)^{2}\right\},
$$

where we put $2 \kappa_{0}^{2}=1$. In (3.5) $\left(\sigma^{A}\right)=\left(\phi^{i}, \varphi^{\alpha}\right) \in \mathbf{R}^{N}$, where $N=n+l$,

$$
\left(\hat{G}_{A B}\right)=\left(\begin{array}{cc}
G_{i j} & 0 \\
0 & C_{\alpha \beta}
\end{array}\right)
$$

is a non-degenerate (block-diagonal) $N \times N$-matrix,

$$
S=S_{e} \equiv \bigsqcup_{a \in \Delta_{e}}\{a\} \times\{e\} \times \Omega_{a, e}
$$


and for $s=(a, e, I) \in S_{e} ; a \in \Delta_{e} ; I \in \Omega_{a, e}$ we denote

$$
\varepsilon_{s}=\theta_{a} \varepsilon(I)= \pm 1
$$

$\Phi^{s}=\Phi^{a, e, I}$ and vectors

$$
L_{s}=\left(L_{A s}\right)=\left(L_{i s}, L_{\alpha s}\right)=\left(l_{i I}, \lambda_{\alpha a}\right) \in \mathbf{R}^{N}
$$

$s \in S_{e}$, are defined by relation

$$
l_{I}=\left(l_{j I}\right) \equiv\left(-\sum_{i \in I} d_{i} \delta_{j}^{i}\right) \in \mathbf{R}^{n}
$$

$i, j=1, \ldots, n ; \alpha=1, \ldots, l ; a \in \Delta_{e}$.

The equations of motion corresponding to (3.5) are

$$
\begin{array}{r}
R_{\mu \nu}\left[g^{0}\right]=\hat{G}_{A B} \partial_{\mu} \sigma^{A} \partial_{\nu} \sigma^{B}+\sum_{s \in S} \varepsilon_{s} e^{2 L_{A s} \sigma^{A}} \partial_{\mu} \Phi^{s} \partial_{\nu} \Phi^{s} \\
\hat{G}_{A B} \triangle\left[g^{0}\right] \sigma^{B}-\sum_{s \in S} \varepsilon_{s} L_{A s} e^{2 L_{B s} \sigma^{B}}\left(\partial \Phi^{s}\right)^{2}=0 \\
\partial_{\mu}\left(\sqrt{\left|g^{0}\right|} g^{0 \mu \nu} e^{2 L_{A s} \sigma^{A}} \partial_{\nu} \Phi^{s}\right)=0
\end{array}
$$

$A=i, \alpha ; i=1, \ldots, n ; \alpha=1, \ldots, l ; s \in S=S_{e}$.

In what follows we define a non-degenerate (real-valued) quadratic form

$$
(X, Y)_{*} \equiv X_{A} \hat{G}^{A B} X_{B}
$$

where $\left(\hat{G}^{A B}\right)=\left(\hat{G}_{A B}\right)^{-1}$.

Proposition 1. Let $S_{*} \subset S$ be a non-empty set of indices such that there exists a set of real non-zero numbers $\nu_{s}, s \in S_{*}$, satisfying the relations

$$
\left(L_{s}, L_{r}\right)_{*}=-\varepsilon_{s}\left(\nu_{s}\right)^{-2} \delta_{s r}
$$

$s, r \in S_{*}$. Let $\left(M_{0}, g^{0}\right)$ be Ricci-flat

$$
R_{\mu \nu}\left[g^{0}\right]=0 .
$$

Then the field configuration

$$
\begin{array}{r}
\sigma^{A}=\sum_{s \in S_{*}} \alpha_{s}^{A} \ln H_{s}, \\
\Phi^{s}=\frac{\nu_{s}}{H_{s}}, \quad s \in S_{*}, \\
\Phi^{s^{\prime}}=C_{s^{\prime}} \in \mathbb{R}, \quad s^{\prime} \in S \backslash S_{*}
\end{array}
$$

satisfies the field equations (3.11)-(3.13) if

$$
\alpha_{s}^{A}=-\hat{G}^{A B} L_{B s} \varepsilon_{s}\left(\nu_{s}\right)^{2},
$$


$A=1, \ldots, N ; s \in S_{*} ; \nu_{s}$ satisfy (3.15), and functions $H_{s}=H_{s}(x)>0$ are harmonic, i.e.

$$
\triangle\left[g^{0}\right] H_{s}=0,
$$

$s \in S_{*}$.

Proposition 1 follows just from substitution of (3.15)-(3.21) into the equations of motion (3.11)-(3.13).

Thus, due to (3.15), the vectors $L_{s}, s \in S_{*}$, are orthogonal to each other, and $\left(L_{s}, L_{s}\right)_{*}$ has a sign opposite to that of $\varepsilon_{s}, s \in S_{*}$. When the form $(\cdot, \cdot)_{*}$ is positive-definite (this take place for $d_{0}>2$ and a positive-definite matrix $C=\left(C_{\alpha \beta}\right)$, the sign is $\varepsilon_{s}=-1$ for all $s \in S_{*}$.

Now, we apply Proposition 1 to the present model with Ricci-flat spaces $\left(M_{i}, g^{i}\right)$, $i=1, \ldots, n$, and zero cosmological constant. From (3.6), (3.9) and (3.14) we get

$$
\left(L_{s}, L_{r}\right)_{*}=<l_{I}, l_{J}>_{*}+\lambda_{a} \cdot \lambda_{b}
$$

with $s=(a, e, I)$ and $r=(b, e, J)$ in $S_{e}\left(a, b \in \Delta_{e} ; I \in \Omega_{a, e} ; J \in \Omega_{b, e}\right)$. Here $l_{I}$ are defined in $(3.10)$ and

$$
\lambda_{a} \cdot \lambda_{b} \equiv C^{\alpha \beta} \lambda_{\alpha a} \lambda_{\beta b}
$$

for $a, b \in \Delta_{e} . \operatorname{In}(3.22)$

$$
<u, v>_{*} \equiv u_{i} G^{i j} v_{j}
$$

is a quadratic form on $\mathbf{R}^{n}$. Here,

$$
G^{i j}=\frac{\delta_{i j}}{d_{i}}+\frac{1}{2-D}
$$

are components of the matrix inverse to the matrix $\left(G_{i j}\right)$ in (3.3).

¿From (3.10), (3.24) and (3.25) we obtain

$$
<l_{I}, l_{J}>_{*}=d(I \cap J)+\frac{d(I) d(J)}{2-D},
$$

$I, J \in \Omega$. In 3.26$) d(\emptyset)=0$. Without restriction let $S_{*}=S_{e}$ (if initially $S_{*} \neq S_{e}$, one may redefine $\Delta_{e}$ and $j_{e}$ from (2.18) such that $S_{*}=S_{e}$ thereafter).

Due to (3.22) and (3.26) the relation (3.15) reads

$$
d(I \cap J)+\frac{d(I) d(J)}{2-D}+C^{\alpha \beta} \lambda_{\alpha a} \lambda_{\beta b}=-\theta_{a} \varepsilon(I)\left(\nu_{a, e, I}\right)^{-2} \delta_{a b} \delta_{I J},
$$

with $I \in \Omega_{a, e}, J \in \Omega_{b, e}, a, b \in \Delta_{e}$, denoting $\nu_{a, e, I} \equiv \nu_{(a, e, I)}$.

For coefficients $\alpha_{s}^{A}$ from (3.20) we get, for $s=(a, e, I) \in S_{e}=S_{*}$,

$$
\begin{array}{r}
\alpha_{s}^{i}=-\theta_{a} G^{i j} l_{j I} \varepsilon(I) \nu_{a, e, I}^{2}=\left(\sum_{j \in I} \delta_{j}^{i}+\frac{d(I)}{2-D}\right) \theta_{a} \varepsilon(I) \nu_{a, e, I}^{2}, \\
\alpha_{I}^{\beta}=-C^{\beta \gamma} \lambda_{\gamma a} \theta_{a} \varepsilon(I) \nu_{a, e, I}^{2},
\end{array}
$$

$i=1, \ldots, n ; \beta, \gamma=1, \ldots, l$. 
Relations (3.17) with $\left(\sigma^{A}\right)=\left(\phi^{i}, \varphi^{\beta}\right), S_{*}=S_{e}$ read

$$
\begin{aligned}
\phi^{i} & =\sum_{s \in S_{e}} \alpha_{s}^{i} \ln H_{s}, \\
\varphi^{\beta} & =\sum_{s \in S_{e}} \alpha_{s}^{\beta} \ln H_{s},
\end{aligned}
$$

$i=1, \ldots, n ; \beta=1, \ldots, l$. These relations imply for $\gamma$ from (3.1)

$$
\gamma=\sum_{s \in S_{e}} \alpha_{s}^{0} \ln H_{s}
$$

where

$$
\alpha_{s}^{0}=\frac{d(I)}{2-D} \theta_{a} \varepsilon(I) \nu_{a, e, I}^{2},
$$

for $s=(a, e, I) \in S_{e}=S_{*}, a \in \Delta_{e} ; I \in \Omega_{a, e}$.

\section{The solution.}

Thus, the equations of motion (2.4)-(2.6) with $\Lambda=0$ defined on the manifold (2.10) have the following solution:

$$
\begin{array}{r}
g=U_{e}\left\{g^{0}+\sum_{i=1}^{n} U_{i, e} g^{i}\right\} \\
U_{e} \equiv\left(\prod_{a \in \Delta_{e}} \prod_{I \in \Omega_{a, e}} H_{a, e, I}^{2 \theta_{a} \varepsilon(I) d(I) \nu_{a, e, I}^{2}}\right)^{\frac{1}{2-D}}, \\
U_{i, e} \equiv \prod_{a \in \Delta_{e}} \prod_{I \in \Omega_{a, e}, I \ni i} H_{a, e, I}^{2 \theta_{a} \varepsilon(I) \nu_{a, e, I}^{2}}, \\
\varphi^{\beta}=\varphi_{e}^{\beta} \equiv-\sum_{a \in \Delta_{e}} \theta_{a} \sum_{I \in \Omega_{a, e}} C^{\beta \gamma} \lambda_{\gamma a} \varepsilon(I) \nu_{a, e, I}^{2} \ln H_{a, e, I}, \\
F^{a}=F^{a, e}=\sum_{I \in \Omega_{a, e}} \nu_{a, e, I} d H_{a, e, I}^{-1} \wedge \tau(I), \quad a \in \Delta_{e}, \\
F^{a}=0, \quad a \notin \Delta_{e}
\end{array}
$$

(we put $\prod_{\emptyset} \ldots \equiv 1$ ), where $\beta=1, \ldots, l ; a \in \Delta_{e}$; forms $\tau(I)$ are defined in (2.15), parameters $\nu_{s} \neq 0$ and $\lambda_{a}$ satisfy the relation (3.27), and functions $H_{s}=H_{s}(x)>0$, are harmonic on $\left(M_{0}, g^{0}\right)$, i.e.

$$
\triangle\left[g^{0}\right] H_{s}=0,
$$

$s \in S_{e} . \operatorname{In}(3.34)$

$$
\operatorname{Ric}\left[g^{0}\right]=\operatorname{Ric}\left[g^{1}\right]=\ldots=\operatorname{Ric}\left[g^{n}\right]=0
$$

( $\operatorname{Ric}\left[g^{\nu}\right]$ is Ricci-tensor corresponding to $g^{\nu}$ ). Relations (3.27) read

$$
\begin{gathered}
-\varepsilon(I) \theta_{a}\left(\nu_{a, e, I}\right)^{-2}=d(I)+\frac{(d(I))^{2}}{2-D}+C^{\alpha \beta} \lambda_{\alpha a} \lambda_{\beta b}, \\
d(I \cap J)+\frac{d(I) d(J)}{2-D}+C^{\alpha \beta} \lambda_{\alpha a} \lambda_{\beta b}=0, \quad(a, I) \neq(b, J),
\end{gathered}
$$


where $I \in \Omega_{a, e} ; J \in \Omega_{b, e} ; a, b \in \Delta_{e}$.

The solution presented here is valid also for $d_{0}=2$. It may be verified using the $\sigma$-model representation (2.32) for $d_{0}=2$ with $f=0$.

Note that, for positive definite matrix $\left(C_{\alpha \beta}\right)$ (or $\left(C^{\alpha \beta}\right)$ ) and $d_{0} \geq 2$, (3.27) implies (cf. [26], Proposition 2)

$$
\varepsilon(I)=-\theta_{a},
$$

for all $I \in \Omega_{a, e} ; a \in \Delta_{e}$. Therefore, for $\theta_{a}=1$ the restriction $g_{\mid M_{I}}$ of the metric (2.11) to a membrane manifold $M_{I}$ has an odd number of linearly independent timelike directions.

However, if the metric $\left(C_{\alpha \beta}\right)$ in the space of scalar fields is not positive-definite (this takes place for $D=12$ model from [49]), then (3.44) may be violated for sufficently negative $\lambda_{a}^{2}=C^{\alpha \beta} \lambda_{\alpha a} \lambda_{\beta b}<0$. In this case a non-trivial potential $A^{a}$ may also exist on an Euclidean p-brane for $\theta_{a}=1$.

\section{Calculations for energy-momentum tensor and ad- ditional constraints}

In this section we omit the restriction (2.31) and show that in general case some additional constraints should be imposed on the $\sigma$-model (2.32).

\subsection{Useful relations}

Let $F_{1}$ and $F_{2}$ be forms of rank $r$ on $(M, g)$ ( $M$ is a manifold and $g$ is a metric on it). We define

$$
\begin{array}{r}
\left(F_{1} \cdot F_{2}\right)_{M N} \equiv\left(F_{1}\right)_{M M_{2} \ldots M_{r}}\left(F_{2}\right)_{N}{ }^{M_{2} \cdots M_{r}} ; \\
F_{1} F_{2} \equiv\left(F_{1} \cdot F_{2}\right)_{M}{ }^{M}=\left(F_{1}\right)_{M_{1} \ldots M_{r}}\left(F_{2}\right)^{M_{1} \ldots M_{r}}
\end{array}
$$

Clearly that

$$
\left(F_{1} \cdot F_{2}\right)_{M N}=\left(F_{2} \cdot F_{1}\right)_{N M}, \quad F_{1} F_{2}=F_{2} F_{1} .
$$

For the form $F^{a, e, I}$ from (2.25) and metric $g$ from (2.11) we obtain

$$
\begin{aligned}
\frac{1}{n_{a} !}\left(F^{a, e, I} \cdot F^{a, e, I}\right)_{\mu \nu} & =\frac{A(I)}{n_{a}} \partial_{\mu} \Phi^{a, e, I} \partial_{\nu} \Phi^{a, e, I} \exp (2 \gamma) ; \\
\frac{1}{n_{a} !}\left(F^{a, e, I} \cdot F^{a, e, I}\right)_{m_{i} n_{i}} & =g_{m_{i} n_{i}}^{i} \frac{A(I)}{n_{a}}\left(\partial \Phi^{a, e, I}\right)^{2} \exp \left(2 \phi^{i}\right),
\end{aligned}
$$

where $i \in I$, indices $m_{i}, n_{i}$ correspond to the manifold $M_{i}$ and

$$
A(I) \equiv A(I, \gamma, \phi)=\varepsilon(I) \exp \left(-2 \gamma-2 \sum_{i \in I} d_{i} \phi^{i}\right),
$$

$I \in \Omega_{a, e}$. All other components of $\left(F^{a, e, I} \cdot F^{a, e, I}\right)_{M N}$ are zero. For the scalar invariant we have

$$
\frac{1}{n_{a} !}\left(F^{a, e, I}\right)^{2} \equiv \frac{1}{n_{a} !} F^{a, e, I} F^{a, e, I}=A(I)\left(\partial \Phi^{a, e, I}\right)^{2},
$$


$I \in \Omega_{a, e}$. We recall that here, as above, we use the notations: $\partial \Phi_{1} \partial \Phi_{2}=g^{0 \mu \nu} \partial_{\mu} \Phi_{1} \partial_{\nu} \Phi_{2}$, $\left(\partial \Phi_{1}\right)^{2}=\partial \Phi_{1} \partial \Phi_{1}$ for functions $\Phi_{1}=\Phi_{1}(x), \Phi_{2}=\Phi_{2}(x)$ on $M_{0}$.

Now consider the tensor field

$$
\left(F^{a, e, I} \cdot F^{a, e, J}\right)_{M N} d z^{M} \otimes d z^{N}
$$

for $I \neq J ; I, J \in \Omega_{a, e}$. From (2.19) we get $d(I)=d(J)$ and hence

$$
I \neq I \cap J ; \quad J \neq I \cap J .
$$

Indeed, if we suppose, for example, that $I \cap J=I$, then we obtain (see (2.17))

$$
d(J)=d(I \cap J)+d(J \backslash I)=d(I)+d(J \backslash I)
$$

or, equivalently, $d(J \backslash I)=0 \Leftrightarrow J=I$. But $I \neq J$.

It may be easily verified that for $I \neq J$ the scalar invariant is trivial:

$$
F^{a, e, I} F^{a, e, J}=0 .
$$

Now we present the non-zero components for the tensor (4.8). Let

$$
w_{1} \equiv\left\{i \mid i \in\{1, \ldots, n\}, d_{i}=1\right\}
$$

The set $w_{1}$ describes all 1-dimensional manifolds among $M_{i}(i \geq 1)$.

It may be verified by a straight-forward calculation that the tensor (4.8) may be nonzero only if

$$
n_{1}=\left|w_{1}\right| \geq 2,
$$

i.e. the number of one-dimensional manifolds among $M_{i}, i \geq 1$, is more than 1 . The only possible non-zero components of (4.8) for $I \neq J ; I, J \in \Omega_{a, e}$, are the following

$$
\begin{aligned}
\frac{1}{\left(n_{a}-1\right) !}\left(F^{a, e, I} \cdot F^{a, e, J}\right)_{1_{i} 1_{j}} & =\delta(i, I \cap J) \delta(j, I \cap J) \varepsilon(I \cap J) \sqrt{\left|g^{i}\right|\left|g^{j}\right|} \\
& \times \exp \left(-2 \gamma-2 \sum_{l \in I \cap J} d_{l} \phi^{l}\right) \partial \Phi^{a, e, I} \partial \Phi^{a, e, J}
\end{aligned}
$$

where $i \neq j ; i, j \in w_{1} ; \delta(i, \mathcal{K})= \pm 1$ is defined for $\{i\} \sqcup \mathcal{K} \in \Omega_{a, e}(i \notin \mathcal{K})$ by the relation

$$
\delta(i, \mathcal{K}) \tau(\{i\} \sqcup \mathcal{K})=\tau_{i} \wedge \tau(\mathcal{K}) .
$$

(The volume form $\tau(I)$ is defined in (2.15).) We put $\tau(\emptyset)=\delta(i, \emptyset)=\varepsilon(\emptyset)=1$. Here and in what follows we denote

$$
(A \sqcup B=C) \Leftrightarrow(A \cup B=C, A \cap B=\emptyset) .
$$




\subsection{Energy-momentum tensor and constraints}

For the "composite" field $F^{a, e}, a \in \Delta_{e}$, from (2.24) we have

$$
\left(F^{a, e}\right)^{2}=\left(\sum_{I \in \Omega_{a, e}} F^{a, e, I}\right)^{2}=\sum_{I \in \Omega_{a, e}}\left(F^{a, e, I}\right)^{2}
$$

and

$$
\left(F^{a, e} \cdot F^{a, e}\right)_{M N}=\sum_{I \in \Omega_{a, e}}\left(F^{a, e, I} \cdot F^{a, e, I}\right)_{M N}+\sum_{\substack{I, J \in \Omega_{a, e} \\ I \neq J}}\left(F^{a, e, I} \cdot F^{a, e, J}\right)_{M N} .
$$

The relations (4.17) and (4.18) imply the following relations for the energy-momentum tensor corresponding to $F^{a, e}$ (see (2.9))

$$
T_{M N}\left[F^{a, e}, g\right]=\sum_{I \in \Omega_{a, e}} T_{M N}\left[F^{a, e, I}, g\right]+\bar{T}_{M N}\left[F^{a, e}, g\right]
$$

where

$$
\bar{T}_{M N}\left[F^{a, e}, g\right] \equiv \frac{1}{\left(n_{a}-1\right) !} \sum_{\substack{I, J \in \Omega_{a, e} \\ I \neq J}}\left(F^{a, e, I} \cdot F^{a, e, J}\right)_{M N} .
$$

Using the results from the previous subsection we obtain that the non-zero components for $\bar{T}_{M N}$ may take place only if the condition (4.13) holds and in this case

$$
\begin{array}{r}
T_{1_{i} 1_{j}}\left[F^{a, e}, g\right]=\bar{T}_{1_{i} 1_{j}}\left[F^{a, e}, g\right] \\
=\sqrt{\left|g^{i}\right|} \sqrt{\left|g^{j}\right|} \exp (-2 \gamma) C_{i j}\left(\Phi^{a, e}, \varphi, \phi, g^{0}\right)
\end{array}
$$

where

$$
\begin{aligned}
C_{i j}=C_{i j}\left(\Phi^{a, o}, \phi, g^{0}\right) \equiv & \sum_{(I, J) \in W_{i j}\left(\Omega_{a, o}\right)} \delta(i, I \cap J) \delta(j, I \cap J) \varepsilon(I \cap J) \\
& \times \exp \left(-2 \sum_{l \in I \cap J} d_{l} \phi^{l}\right) g^{0 \mu \nu} \partial_{\mu} \Phi^{a, o, I} \partial_{\nu} \Phi^{a, o, J}
\end{aligned}
$$

$a \in \Delta_{o} ; i, j \in w_{1} ; i \neq j\left(\left|w_{1}\right| \geq 2\right) \Phi^{a, o}=\left(\Phi^{a, o, I}, I \in \Omega_{a, o}\right)$. Here $o=e$ and

$$
W_{i j}\left(\Omega_{1}\right) \equiv\left\{(I, J) \mid I, J \in \Omega_{1}, I=\{i\} \sqcup(I \cap J), J=\{j\} \sqcup(I \cap J)\right\} .
$$

$i, j \in w_{1}, i \neq j, \Omega_{1} \subset \Omega$.

The non-block-diagonal part of the total energy-momentum tensor (2.7) has the following form

$$
T_{1_{i} 1_{j}}\left[F^{e}, \varphi, g\right]=\sqrt{\left|g^{i}\right|} \sqrt{\left|g^{j}\right|} \mathrm{e}^{-2 \gamma} C_{i j}^{e}\left(\Phi^{e}, \varphi, \phi, g^{0}\right),
$$

where $\Phi^{e}=\left(\Phi^{a, e}\right)$ and

$$
C_{i j}^{e}\left(\Phi^{e}, \varphi, \phi, g^{0}\right) \equiv \sum_{a \in \Delta_{e}} \theta_{a} \exp \left[2 \lambda_{a}(\varphi)\right] C_{i j}\left(\Phi^{a, e}, \phi, g^{0}\right)
$$

$i, j \in w_{1} ; i \neq j$. Relations (4.24), (4.25) follow from relations (2.7), (4.21) and (4.22). 
¿From Einstein-Hilbert equations (2.4) $(\Lambda=0)$ and block-diagonal form of metric and Ricci-tensor (in the considered ansatz) it follows that

$$
T_{1_{i} 1_{j}}=0
$$

$i \neq j$, and hence

$$
C_{i j}^{e}\left(\Phi^{e}, \varphi, \phi, g^{0}\right)=0, \quad i<j
$$

$i, j \in w_{1}\left(C_{i j}^{e}=C_{j i}^{e}\right)$.

Thus, we obtain

$$
m_{1}=n_{1}\left(n_{1}-1\right) / 2
$$

constraints, where $n_{1}=\left|w_{1}\right| \geq 2$ is the number of 1-dimensional manifolds among $M_{i}(i \geq$ 1). The equation of motions (2.5), (2.6) and block-diagonal part of (2.4) are equivalent to the equations of motion for the $\sigma$-model (2.32) (or (3.2) when the harmonic gauge is fixed). The non-block-diagonal part of (2.4) leads to $m_{1}$ constraints on the fields of $\sigma$-model (2.32) (or (3.2)).

Restriction 2e. It follows from the presented above consideration that the constraints are absent or are identically satisfied in the following two cases: i) $n_{1} \leq 1$ (2.31); ii) $n_{1}>1$ and

$$
W_{i j}\left(\Omega_{a, e}\right)=\emptyset,
$$

(see (4.23) $i<j ; i, j \in w_{1}, a \in \Delta_{e}$. The condition (4.29) means that for any $a \in \Delta_{e}$ and $i<j(i, j=1, \ldots, n)$ such that $d_{i}=d_{j}=1$, there are no sets $I, J \in \Omega_{a, e}$, such that $I=\{i\} \sqcup(I \cap J)$ and $J=\{j\} \sqcup(I \cap J)$. Literally (or physically) speaking the $p$-branes "feel" the presence of additional (internal) 1-dimensional directions (for example, times). The non-trivial constraints in the world with several times occur, when there are at least two $p$-branes (with the same $p$ ) "living" in different "times" and charged by the same field of form $F^{a}\left(a \in \Delta_{e}\right)$.

Thus, we weakened the Restriction 1 (2.31) by adding the additional restrictions on $p$-branes (4.29). The exact solutions of Sect. 3 also take place for the more general case satisfying Restriction 2e.

\section{$5 \quad$ Magnetic p-branes}

Here we consider the ansatz for magnetically charged $p$-branes that is dual to the one considered in subsection 2.1.

\subsection{Ansatz for composite magnetic $p$-branes}

Let $\Delta_{m} \subset \Delta$ be a non-empty subset and

$$
\begin{aligned}
j_{m} & : \Delta_{m} \rightarrow P_{*}(\Omega) \\
& a \mapsto \Omega_{a, m} \in \Omega, \quad \Omega_{a, m} \neq \emptyset
\end{aligned}
$$

is a map satisfying the condition

$$
n_{a}=D-d(I)-1
$$


for all $I \in \Omega_{a m} ; a \in \Delta_{m}$.

For the potential forms $A^{a}, a \in \Delta_{m}$, we make the ansatz

$$
\begin{array}{r}
A^{a}=A^{a, m}=0, \quad a \in \Delta \backslash \Delta_{m}, \\
A^{a}=A^{a, m}=\sum_{I \in \Omega_{a, m}} A^{a, m, I}, \quad a \in \Delta_{m},
\end{array}
$$

where

$$
F^{a, m, I} \equiv d A^{a, m, I}=\mathrm{e}^{-2 \lambda_{a}(\varphi)} *\left(d \Phi^{a, m, I} \wedge \tau(I)\right),
$$

and $\Phi^{a, m, I}: M_{0} \rightarrow \mathbf{R}$ are smooth functions on $M_{0}$ and volume forms $\tau(I)$ are defined in (2.15), $I \in \Omega_{a, m} ; a \in \Delta_{m}$. In (5.5) $*=*[g]$ is Hodge operator on $(M, g)$.

¿From (5.5) Bianchi identities follow

$$
d F^{a, m, I}=0
$$

$a \in \Delta_{m} ; I \in \Omega_{a, m}$.

In general case relations (5.6) guarantee (at least) the local existence of forms $A^{a, m, I}$ satisfying (5.5).

The field equations (2.6) corresponding to $A^{a, m}$ (generalized Maxwell equations) written in the equivalent form

$$
* d *\left(\mathrm{e}^{2 \lambda_{a}(\varphi)} F^{a, m}\right)=0
$$

are satisfied identically for the ansatz (5.3)-(5.5)).

Now we impose, as in Section 2, Restriction 1. Then it may be verified that field equations (2.4), (2.5) and Bianchi relations (5.6) may be obtained as the equations of motion for the $\sigma$-model with the action (2.32) and

$$
\mathcal{L}_{A}=\mathcal{L}_{A, m} \equiv-\varepsilon_{g} \sum_{a \in \Delta_{m}} \theta_{a} \sum_{I \in \Omega_{a, m}} \varepsilon(I) \exp \left(-2 \lambda_{a}(\varphi)-2 \sum_{i \in I} d_{i} \phi^{i}\right)\left(\partial \Phi^{a, m, I}\right)^{2},
$$

where $\varepsilon_{g} \equiv \operatorname{sign} \operatorname{det}\left(g_{M N}\right)$.

It should be noted that the sign in (5.8) is opposite to that obtained by a straightforward substitution of (5.3)-(5.5) into action (2.1). This follows from the relation (2.42) and the following identity

$$
\frac{1}{k !} F^{2}=\frac{\varepsilon_{g}}{k_{*} !}(* F)^{2},
$$

where $k=\operatorname{rank} F, k_{*}=\operatorname{rank}(* F)=D-k$. The reason for the appearance of the additional sign "-" in (5.8) can be easily explained by using the following relation for energy-momentum tensor

$$
T_{M N}[* F, g]=-\varepsilon_{g} T_{M N}[F, g] .
$$

Relations (5.9) and (5.10) follow from the formulas

$$
\begin{array}{r}
\frac{1}{k_{*} !}\left(* F_{1}\right)\left(* F_{2}\right)=\frac{\varepsilon_{g}}{k !} F_{1} F_{2} ; \\
\frac{1}{\left(k_{*}-1\right) !}\left[\left(* F_{1}\right) \cdot\left(* F_{2}\right)\right]_{M N}=\frac{\varepsilon_{g}}{k !}\left\{g_{M N}\left(F_{1} F_{2}\right)-k\left(F_{2} \cdot F_{1}\right)_{M N}\right\},
\end{array}
$$

where $k=\operatorname{rank} F_{i}, k_{*}=\operatorname{rank}\left(* F_{i}\right), i=1,2$. 


\subsection{Exact solutions}

For $d_{0} \neq 2, \xi_{i}=\Lambda=0, i=1, \ldots, n$, as in pure electric case we are led to the $\sigma$-model (3.5) (we consider the harmonic gauge (3.1)) with the set

$$
S=S_{m} \equiv \bigsqcup_{a \in \Delta_{m}}\{a\} \times\{m\} \times \Omega_{a, m},
$$

and for $s=(a, m, I) \in S_{m}, I \in \Omega_{a, m}, a \in \Delta_{m}$

$$
\varepsilon_{s}=-\varepsilon_{g} \theta_{a} \varepsilon(I)= \pm 1
$$

$\Phi^{s}=\Phi^{a, m, I}$ and

$$
L_{s}=\left(L_{A s}\right)=\left(L_{i s}, L_{\alpha s}\right)=\left(l_{i I},-\lambda_{\alpha a}\right) \in \mathbf{R}^{N},
$$

$s \in S_{m}$, with $l_{j I}$ defined in (3.10).

Applying Proposition 1 for the considered values of $S=S_{m}=S_{*}, \varepsilon_{s}$ and $L_{s}$ from (5.13)-(5.15) respectively we get the "magnetic" analog of the "electric" solutions (3.34)(3.39)

$$
\begin{array}{r}
g=U_{m}\left\{g^{0}+\sum_{i=1}^{n} U_{i, m} g^{i}\right\}, \\
U_{m} \equiv\left(\prod_{a \in \Delta_{m}} \prod_{I \in \Omega_{a, m}} H_{a, m, I}^{-2 \varepsilon_{g} \theta_{a} \varepsilon(I) d(I) \nu_{a, m, I}^{2}}\right)^{\frac{1}{2-D}}, \\
U_{i, m} \equiv \prod_{a \in \Delta_{m}} \prod_{I \in \Omega_{a, m}, I \ni i} H_{a, m, I}^{-2 \varepsilon_{g} \theta_{a} \varepsilon(I) \nu_{a, m, I}^{2},} \\
\varphi^{\beta}=\varphi_{m}^{\beta} \equiv-\varepsilon_{g} \sum_{a \in \Delta_{m}} \theta_{a} \sum_{I \in \Omega_{a, m}} \lambda_{a}^{\beta} \varepsilon(I) \nu_{a, m, I}^{2} \ln H_{a, m, I}, \\
F^{a}=F^{a, m}=\sum_{I \in \Omega_{a, m}} \nu_{a, m, I}\left(*_{0} d H_{a, m, I}\right) \wedge \tau(\bar{I}), \quad a \in \Delta_{m}, \\
F^{a}=0, \quad a \in \Delta \backslash \Delta_{m},
\end{array}
$$

where $i=1, \ldots, n ; H_{a, m, I}=H_{a, m, I}(x)>0$ are harmonic functions on $\left(M_{0}, g^{0}\right)$

$$
\Delta\left[g^{0}\right] H_{a, m, I}=0,
$$

and parameters satisfy the relations

$$
\begin{array}{r}
\varepsilon_{g} \varepsilon(I) \theta_{a} \nu_{a, m, I}^{-2}=d(I)+\frac{(d(I))^{2}}{2-D}+C^{\alpha \beta} \lambda_{\alpha a} \lambda_{\beta b}, \\
d(I \cap J)+\frac{d(I) d(J)}{2-D}+C^{\alpha \beta} \lambda_{\alpha a} \lambda_{\beta b}=0, \quad(a, I) \neq(b, J),
\end{array}
$$

$a, b \in \Delta_{m} ; I \in \Omega_{a, m} ; J \in \Omega_{b, m}$.

In $(5.20)$

$$
\bar{I} \equiv I_{0} \backslash I, \quad I_{0} \equiv\{1, \ldots, n\}
$$

is "dual" set, and $*_{0} d \Phi$ is the Hodge dual form on $\left(M_{0}, g^{0}\right)\left(*_{0}=*\left[g^{0}\right]\right)$. 
The relation (5.20) follows from the formula (see (5.5))

$$
F^{a, m, I}=\varepsilon(I) \mu(I) \exp \left(f-2 \sum_{i \in I} d_{i} \phi^{i}-2 \lambda_{a}(\varphi)\right)\left(*_{0} d \Phi^{a, m, I}\right) \wedge \tau(\bar{I}),
$$

$f=0$ and the relation

$$
\exp \left(-2 \lambda_{a}(\varphi)-2 \sum_{i \in I} d_{i} \phi^{i}\right)=H_{a, m, I}^{2}
$$

In (5.26) $\mu(I)= \pm 1$ is defined by relation

$$
\mu(I) d x^{\mu} \wedge \tau\left(I_{0}\right)=\tau(\bar{I}) \wedge d x^{\mu} \wedge \tau(I)
$$

Here we define $\nu_{a, m, I}=-\varepsilon(I) \mu(I) \nu_{s}, s=(a, m, I)$. The relation (5.27) is a special case of a more general identity for the solutions satisfying Proposition 1:

$$
\exp \left(2 L_{A s} \sigma^{A}\right)=H_{s}^{2}, \quad s \in S_{*} .
$$

Note that, for positive definite matrix $\left(C_{\alpha \beta}\right)$ and $d_{0} \geq 2$, equation (5.23) implies

$$
\varepsilon(I)=\theta_{a} \varepsilon_{g}
$$

for $I \in \Omega_{a, m} ; a \in \Delta_{m}$. So, for $\theta_{a}=1$ Euclidean magnetically charged $p$-branes "may live" in space-times with even number of time directions.

\subsection{Energy-momentum tensor and constraints}

We rewrite the relation (5.5)

$$
F^{a, m, I}=\mathrm{e}^{-2 \lambda_{a}(\varphi)} * \hat{F}^{a, m, I}, \quad \hat{F}^{a, m, I}=d \Phi^{a, m, I} \wedge \tau(I),
$$

$a \in \Delta_{m}$. Analogously to 4.11$)$ we get

$$
\hat{F}^{a, m, I} \hat{F}^{a, m, J}=0 \Longrightarrow F^{a, m, I} F^{a, m, J}=0
$$

for $I \neq J ; I, J \in \Omega_{a, m}$ (see (5.11)).

For the "composite" field $F^{a, m}=d A^{a, m}, a \in \Delta_{m}$, satisfying (5.4) and (5.5) we have (see $(5.32))$

$$
\left(F^{a, m}\right)^{2}=\left(\sum_{I \in \Omega_{a, m}} F^{a, m, I}\right)^{2}=\sum_{I \in \Omega_{a, m}}\left(F^{a, m, I}\right)^{2}
$$

and

$$
\left(F^{a, m} \cdot F^{a, m}\right)_{M N}=\sum_{I \in \Omega_{a, m}}\left(F^{a, m, I} \cdot F^{a, m, I}\right)_{M N}+\sum_{\substack{I, J \in \Omega_{a}, m \\ I \neq J}}\left(F^{a, m, I} \cdot F^{a, m, J}\right)_{M N} .
$$

The relations (5.33) and (5.34) imply the following relations for the energy-momentum tensor corresponding to $F^{a, m}$ (see (2.9))

$$
T_{M N}\left[F^{a, m}, g\right]=\sum_{I \in \Omega_{a, m}} T_{M N}\left[F^{a, m, I}, g\right]+\bar{T}_{M N}\left[F^{a, m}, g\right],
$$


where

$$
\bar{T}_{M N}\left[F^{a, m}, g\right] \equiv \frac{1}{\left(n_{a}-1\right) !} \sum_{\substack{I, J \in \Omega_{a}, m \\ I \neq J}}\left(F^{a, m, I} \cdot F^{a, m, J}\right)_{M N} .
$$

Analogously to (4.19), (4.20) we get

$$
T_{M N}\left[\hat{F}^{a, m}, g\right]=\sum_{I \in \Omega_{a, m}} T_{M N}\left[\hat{F}^{a, m, I}, g\right]+\bar{T}_{M N}\left[\hat{F}^{a, m}, g\right],
$$

where

$$
\bar{T}_{M N}\left[\hat{F}^{a, m}, g\right] \equiv \frac{1}{\left(n_{a}^{*}-1\right) !} \sum_{\substack{I, J \in \Omega_{a}, m \\ I \neq J}}\left(\hat{F}^{a, m, I} \cdot \hat{F}^{a, m, J}\right)_{M N},
$$

where $n_{a}^{*}=D-n_{a}$ and $a \in \Delta_{m}$. Here

$$
\begin{array}{r}
\hat{F}^{a, m}=0, \quad a \in \Delta \backslash \Delta_{m}, \\
\hat{F}^{a, m}=\sum_{I \in \Omega_{a, m}} \hat{F}^{a, m, I}, \quad a \in \Delta_{m} .
\end{array}
$$

It is clear that

$$
F^{a, m}=\mathrm{e}^{-2 \lambda_{a}(\varphi)} * \hat{F}^{a, m} .
$$

¿From (5.10), (5.35)-(5.41) we get

$$
\begin{aligned}
T_{M N}\left[F^{a, m}, g\right] & =-\varepsilon_{g} \mathrm{e}^{-4 \lambda_{a}(\varphi)} T_{M N}\left[\hat{F}^{a, m}, g\right], \\
\bar{T}_{M N}\left[F^{a, m}, g\right] & =-\varepsilon_{g} \mathrm{e}^{-4 \lambda_{a}(\varphi)} \bar{T}_{M N}\left[\hat{F}^{a, m}, g\right] .
\end{aligned}
$$

Using the results from the previous section applied to $T_{M N}\left[\hat{F}^{a, m}, g\right]$ and $(5.42),(5.43)$ we obtain that the non-zero components for $\bar{T}_{M N}$ may take place only if the condition (4.13) holds and in this case

$$
\begin{array}{r}
T_{1_{i} 1_{j}}\left[F^{a, m}, g\right]=\bar{T}_{1_{i} 1_{j}}\left[F^{a, m}, g\right] \\
=\sqrt{\left|g^{i}\right|} \sqrt{\left|g^{j}\right|} \exp \left(-2 \gamma-4 \lambda_{a}(\varphi)\right)\left(-\varepsilon_{g}\right) C_{i j}\left(\Phi^{a, m}, \phi, g^{0}\right),
\end{array}
$$

where $C_{i j}\left(\Phi^{a, m}, \phi, g^{0}\right)$ is defined in (4.22) (with $o=m$ ).

The non-block-diagonal part of the total energy-momentum tensor (2.7) (in magnetic case) has the following form

$$
T_{1_{i} 1_{j}}\left[F^{m}, \varphi, g\right]=\sqrt{\left|g^{i}\right|} \sqrt{\left|g^{j}\right|} \mathrm{e}^{-2 \gamma} C_{i j}^{m}\left(\Phi^{m}, \varphi, \phi, g^{0}\right)
$$

where $\Phi^{m}=\left(\Phi^{a, m}\right)$ and

$$
C_{i j}^{m}\left(\Phi^{m}, \varphi, \phi, g^{0}\right) \equiv\left(-\varepsilon_{g}\right) \sum_{a \in \Delta_{m}} \theta_{a} \exp \left[-2 \lambda_{a}(\varphi)\right] C_{i j}\left(\Phi^{a, m}, \phi, g^{0}\right),
$$

$i, j \in w_{1} ; i \neq j$. Relations (5.45), (5.46) follows from relations (2.7), (5.44).

¿From Einstein-Hilbert equations (2.4) $(\Lambda=0)$, block-diagonal form of metric and Ricci-tensor (in the considered ansatz) and (5.45) we get the magnetic analog of constraints (4.27)

$$
C_{i j}^{m}\left(\Phi^{m}, \varphi, \phi, g^{0}\right)=0, \quad i<j
$$


$i, j \in w_{1}$.

Restriction $\mathbf{2 m}$. The constraints are satisfied identically in the case $n_{1} \leq 1$ (2.31) or when $n_{1}>1$ and

$$
W_{i j}\left(\Omega_{a, m}\right)=\emptyset
$$

$i<j ; i, j \in w_{1} ; a \in \Delta_{m}$.

\section{Electro-magnetic case}

Now we consider the "superposition" of the ansätze from Sections 4 and 5, i.e. we put

$$
\begin{array}{r}
F^{a}=0, \quad a \in \Delta \backslash\left(\Delta_{e} \cup \Delta_{m}\right), \\
F^{a}=F^{a, e}=\sum_{I \in \Omega_{a, e}} F^{a, e, I}, \quad a \in \Delta_{e} \backslash \Delta_{m}, \\
F^{a}=F^{a, m}=\sum_{I \in \Omega_{a, m}} F^{a, m, I}, \quad a \in \Delta_{m} \backslash \Delta_{e}, \\
F^{a}=F^{a, e}+F^{a, m}=\sum_{I \in \Omega_{a, e}} F^{a, e, I}+\sum_{J \in \Omega_{a, m}} F^{a, m, J}, \quad a \in \Delta_{e} \cap \Delta_{m},
\end{array}
$$

where $F^{a, e, I}$ and $F^{a, m, J}$ are defined in (2.25) and (5.5) respectively.

\subsection{Energy-momentum tensor and constraints}

Let $d_{0} \neq 2$. For $a \in \Delta_{e} \cap \Delta_{m}$ we obtain

$$
\left(* \hat{F}^{a, m, I}\right) F^{a, e, J}=F^{a, e, J}\left(* \hat{F}^{a, m, I}\right)=0,
$$

$I \in \Omega_{a, m} ; J \in \Omega_{a, e} ;$ and hence

$$
F^{a, e} F^{a, m}=F^{a, m} F^{a, e}=0 .
$$

Relation (6.5) is due to the non-equal numbers of $M_{0}$ indices in the non-zero components of forms $\hat{F}$ and $F$ in (6.5). From (6.6) we get

$$
\left(F^{a}\right)^{2}=\left(F^{a, e}\right)^{2}+\left(F^{a, m}\right)^{2},
$$

where $\left(F^{a, e}\right)^{2}$ and $\left(F^{a, m}\right)^{2}$ are expressed by (4.17) and (5.33). We also get

$$
\begin{aligned}
\left(F^{a} \cdot F^{a}\right)_{M N} & =\left(F^{a, e} \cdot F^{a, e}\right)_{M N}+\left(F^{a, m} \cdot F^{a, m}\right)_{M N} \\
& +\left(F^{a, e} \cdot F^{a, m}\right)_{M N}+\left(F^{a, m} \cdot F^{a, e}\right)_{M N}
\end{aligned}
$$

for $a \in \Delta_{e} \cap \Delta_{m}$.

The relations (4.17), (5.33), (6.7), 6.8) imply

$$
T_{M N}\left[F^{a}, g\right]=T_{M N}\left[F^{a, e}, g\right]+T_{M N}\left[F^{a, m}, g\right]+\tilde{T}_{M N}\left[F^{a, e}, F^{a, m}, g\right]
$$

where $T_{M N}\left[F^{a, e}, g\right], T_{M N}\left[F^{a, m}, g\right]$ are presented in (4.19), (5.35) respectively and

$$
\tilde{T}_{M N}^{a}=\tilde{T}_{M N}\left[F^{a, e}, F^{a, m}, g\right] \equiv \frac{1}{\left(n_{a}-1\right) !}\left\{\left(F^{a, e} \cdot F^{a, m}\right)_{M N}+\left(F^{a, m} \cdot F^{a, e}\right)_{M N}\right\},
$$


$a \in \Delta_{e} \cap \Delta_{m}$. We obtain

$$
=\sum_{I \in \Omega_{a, m}} \sum_{J \in \Omega_{a, e}}^{\left(F^{a, m} \cdot F^{a, e}\right)_{M N}=\left(F^{a, e} \cdot F^{a, m}\right)_{N M}}
$$

$a \in \Delta_{e} \cap \Delta_{m}$.

The tensor (6.10) is trivial for $d_{0}>3$ and may have non-zero (non-diagonal) components for $d_{0}=1,3$, when $n_{1}=\left|w_{1}\right| \geq 1$ (i.e. there are 1-dimensional $M_{i}$ ).

Indeed, calculations give (for $d_{0} \neq 2$ ) that

$$
\left[\left(* \hat{F}^{a, m, I}\right) \cdot F^{a, e, J}\right]_{M N}=\left[F^{a, e, J} \cdot\left(* \hat{F}^{a, m, I}\right)\right]_{N M}
$$

may have non-zero components only if $d_{0}=1 ; \bar{I}=\{j\} \sqcup J, j \in w_{1}$ :

$$
\begin{array}{r}
{\left[\left(* \hat{F}^{a, m, I}\right) \cdot F^{a, e, J}\right]_{1_{j} \mu}=\left[F^{a, e, J} \cdot\left(* \hat{F}^{a, m, I}\right)\right]_{\mu 1_{j}}=d(J) ! \varepsilon_{g} \mu(I) \delta(j, J)\left|g^{j}\right|^{1 / 2}} \\
\times \exp \left(-3 \gamma-\sum_{i=1}^{n} d_{i} \phi^{i}+2 \phi^{j}\right)\left|g^{0}\right|^{-1 / 2} \partial_{1_{0}} \Phi^{a, m, I} \partial_{\mu} \Phi^{a, e, J}
\end{array}
$$

or $d_{0}=3 ; J=\{i\} \sqcup \bar{I}, i \in w_{1}$

$$
\begin{aligned}
{\left[\left(* \hat{F}^{a, m, I}\right)\right.} & \left.\cdot F^{a, e, J}\right]_{\mu 1_{i}}=\left[F^{a, e, J} \cdot\left(* \hat{F}^{a, m, I}\right)\right]_{1_{i} \mu}=\varepsilon_{g_{0}} \varepsilon_{g} \delta(i, I) \mu(I) d(J) ! \\
& \times\left|g^{i}\right|^{1 / 2}\left(\left|g^{0}\right|^{1 / 2} \varepsilon_{\mu \rho \nu} \nabla^{\rho} \Phi^{a, m, I} \nabla^{\nu} \Phi^{a, e, J}\right) \exp \left(-\gamma-\sum_{i=1}^{n} d_{i} \phi^{i}\right) .
\end{aligned}
$$

Recall that $w_{1}$ is defined in (4.12).

¿From (6.9)-(6.14) we have for $d_{0}=1$

$$
T_{1_{j} 1_{0}}=\varepsilon_{g}\left|g^{j}\right|^{1 / 2}\left|g^{0}\right|^{-1 / 2} \exp \left(-3 \gamma-\sum_{i=1}^{n} d_{i} \phi^{i}+2 \phi^{j}\right) C_{j 1_{0}}^{(1)}\left(\Phi^{e}, \Phi^{m}\right),
$$

where

$$
C_{j 1_{0}}^{(1)}\left(\Phi^{e}, \Phi^{m}\right) \equiv \sum_{a \in \Delta_{e} \cap \Delta_{m}} \sum_{(I, J) \in W_{j}^{(1)}} \mu(I) \delta(j, J) \partial_{1_{0}} \Phi^{a, m, I} \partial_{1_{0}} \Phi^{a, e, J},
$$

and

$$
W_{j}^{(1)}=W_{j}^{(1)}\left(\Omega_{a, m}, \Omega_{a, e}\right) \equiv\left\{(I, J) \in \Omega_{a, m} \times \Omega_{a, e} \mid \bar{I}=\{j\} \sqcup J\right\},
$$

$j \in w_{1}$. For $d_{0}=3$ the analogous relations read

$$
\begin{array}{r}
T_{\mu 1_{i}}=\varepsilon_{g 0} \varepsilon_{g}\left|g^{i}\right|^{1 / 2} \exp \left(-\gamma-\sum_{i=1}^{n} d_{i} \phi^{i}\right) C_{i \mu}^{(3)}\left(\Phi^{e}, \Phi^{m}, g^{0}\right) ; \\
C_{i \mu}^{(3)}\left(\Phi^{e}, \Phi^{m}, g^{0}\right) \equiv \sum_{a \in \Delta_{e} \cap \Delta_{m}} \sum_{(I, J) \in W_{i}^{(3)}} \delta(i, I) \mu(I)\left|g^{0}\right|^{1 / 2} \\
\times \varepsilon_{\mu \rho \nu} \nabla^{\rho} \Phi^{a, m, I} \nabla^{\nu} \Phi^{a, e, J} ; \\
W_{i}^{(3)}=W_{i}^{(3)}\left(\Omega_{a, m}, \Omega_{a, e}\right) \equiv\left\{(I, J) \in \Omega_{a, m} \times \Omega_{a, e} \mid J=\{i\} \sqcup \bar{I}\right\},
\end{array}
$$


$i \in w_{1}$ and $\mu=1_{0}, 2_{0}, 3_{0}$.

Thus, in the "electro-magnetic" case for $d_{0}=1,3$ we are led to $n_{1}=\left|w_{1}\right|$ additional constraints

$$
C_{i \mu}^{\left(d_{0}\right)}\left(\Phi^{e}, \Phi^{m}, g^{0}\right)=0
$$

$i \in w_{1}$ and $\mu=1_{0}, \ldots,\left(d_{0}\right)_{0}$.

As to $T_{1_{i} 1_{j}}$ - components for $i, j \in w_{1}$ we get from (6.9), (4.21), (4.22), (5.44)

$$
T_{1_{i} 1_{j}}=\sqrt{\left|g^{i}\right|} \sqrt{\left|g^{j}\right|} \mathrm{e}^{-2 \gamma} C_{i j}\left(\Phi^{e}, \Phi^{m}, \varphi, \phi, g^{0}\right),
$$

where

$$
\begin{array}{r}
C_{i j}\left(\Phi^{e}, \Phi^{m}, \varphi, \phi, g^{0}\right)=\sum_{a \in \Delta_{e}} \theta_{a} \exp \left[2 \lambda_{a}(\varphi)\right] C_{i j}\left(\Phi^{a, e}, \phi, g^{0}\right) \\
-\varepsilon_{g} \sum_{a \in \Delta_{m}} \theta_{a} \exp \left[-2 \lambda_{a}(\varphi)\right] C_{i j}\left(\Phi^{a, m}, \phi, g^{0}\right)
\end{array}
$$

$(\operatorname{see}(4.22))$.

Thus, for $n_{1} \geq 2$ the constraints (4.27), (5.47) are generalized as follows

$$
C_{i j}\left(\Phi^{e}, \Phi^{m}, \varphi, \phi, g^{0}\right)=0, \quad i<j
$$

$i, j \in w_{1}$.

Now let us consider the case $d_{0}=2$. It may be verified that all the presented in this section relations are unchanged if the following restrictions are imposed:

$$
\begin{gathered}
W\left(\Omega_{a, e}, \hat{\Omega}_{a, m}\right)=\emptyset, \\
W_{i j}\left(\Omega_{a, e}, \hat{\Omega}_{a, m}\right)=\emptyset,
\end{gathered}
$$

where $i \neq j ; i, j \in w_{1}, a \in \Delta_{e} \cap \Delta_{m}$ and

$$
\begin{array}{r}
\hat{\Omega}_{a, m}=\left\{I \in \Omega \mid \bar{I} \in \Omega_{a, m}\right\}, \\
W\left(\Omega_{1}, \Omega_{2}\right) \equiv\left\{\left(I_{1}, I_{2}\right) \in \Omega_{1} \times \Omega_{2} \mid I_{1}=I_{2}\right\}, \\
W_{i j}\left(\Omega_{1}, \Omega_{2}\right) \equiv\left\{\left(I_{1}, I_{2}\right) \in \Omega_{1} \times \Omega_{2} \mid I_{1}=\{i\} \sqcup\left(I_{1} \cap I_{2}\right), I_{2}=\{j\} \sqcup\left(I_{1} \cap I_{2}\right)\right\} .
\end{array}
$$

\section{$6.2 \sigma$-model}

In the general case we are led to the $\sigma$-model (2.32)-(2.34) with

$$
\mathcal{L}_{A}=\mathcal{L}_{A, e}+\mathcal{L}_{A, m}
$$

where $\mathcal{L}_{A, e}$ and $\mathcal{L}_{A, m}$ are defined in (2.35) and (5.8) respectively. We also obtain constraints (6.24) for $n_{1} \geq 2, d_{0} \neq 2$ and (6.21) for $n_{1} \geq 1$ for $d_{0}=1,3$. For $d_{0}=2$ the constraints are the same if the restrictions (6.25), (6.26) hold.

We recall that all the constraints occur due to non-block-diagonal part of energymomentum tensor. The block-diagonal part gives rise to $\sigma$-model itself.

Thus we are led to the following 
Proposition 2. Let us consider the model (2.1) where the manifold, metric, scalar fields and forms are defined by relations (2.10), (2.11)-(2.12), (2.26) and (6.1)-(6.4) respectively. Then for $d_{0} \neq 2$ and $\gamma=\gamma_{0}(\phi)$ from (3.1) the equations of motion (2.4)-(2.6) and Bianchi identities (5.6) are equivalent to the equations of motion for the $\sigma$-model (3.2)-(3.4) with the Lagrangians $\mathcal{L}, \mathcal{L}_{\varphi}$ from (2.33), 2.34) and $\mathcal{L}_{A}$ from (6.30) (see also (2.35) and (5.8) ) and the constraints (6.24) (for all $d_{0} \neq 2$ ) and (6.21) (for $d_{0}=1,3$ ) imposed.

Proof. The appearance of constraints was verified above. Now we consider the reduction to $\sigma$-model itself. For $(F, \varphi)$-part of field equations and Bianchi identities the equivalence with corresponding equations of motion for $\sigma$-model can be readily verified. Here we consider the Einstein equations (2.4) written in the form

$$
R_{M N}=Z_{M N}+\frac{2 \Lambda}{D-2} g_{M N}
$$

where

$$
Z_{M N} \equiv T_{M N}+\frac{T}{2-D} g_{M N}
$$

and $T=T_{M}^{M}$. Here

$$
Z_{M N}=Z_{M N}[\varphi]+\sum_{a \in \Delta} \theta_{a} e^{2 \lambda_{a}(\varphi)} Z_{M N}\left[F^{a}, g\right]
$$

where

$$
\begin{gathered}
Z_{M N}[\varphi]=C_{\alpha \beta} \partial_{M} \varphi^{\alpha} \partial_{N} \varphi^{\beta} \\
Z_{M N}\left[F^{a}, g\right]=\frac{1}{n_{a} !}\left[\frac{n_{a}-1}{2-D} g_{M N}\left(F^{a}\right)^{2}+n_{a} F_{M M_{2} \ldots M_{n_{a}}}^{a} F_{N}^{a, M_{2} \ldots M_{n_{a}}}\right]
\end{gathered}
$$

For block-diagonal part of (6.35) we have ¿from (6.7) and (6.8) (see also (4.17), 4.18$),(5.33)$ and (5.34))

$$
Z_{M N}\left[F^{a}, g\right]=\sum_{I \in \Omega_{a, e}} Z_{M N}\left[F^{a, e, I}, g\right]+\sum_{J \in \Omega_{a, m}} Z_{M N}\left[F^{a, m, J}, g\right]
$$

where $(M, N)=(\mu, \nu),\left(m_{i}, n_{i}\right) ; i=1, \ldots, n\left(F^{a, e, I}\right.$ and $F^{a, m, J}$ are defined in (2.25) and (5.5) respectively). Here we put $\Omega_{a, e}=\emptyset$ and $\Omega_{b, m}=\emptyset$ for $a \notin \Delta_{e}$ and $b \notin \Delta_{m}$ respectively. Using the relations for Ricci tensor (2.27), (2.28) with $\gamma=\gamma_{0}(\phi)$ from (3.1) and relations (2.42), (4.4), (4.5), (5.11), (5.12) and (6.4) we obtain that $\left(m_{i}, n_{i}\right)$-components of Einstein equations (6.31) $(i=1, \ldots, n)$ are equivalent to $\phi^{i}$-part of $\sigma$-model equations and $(\mu, \nu)$ components of Einstein equations (6.31) are equivalent to $g^{0}$-part of $\sigma$-model equations of motion (or $\sigma$-model Einstein equations). Note that dealing with $(\mu, \nu)$-components of (6.31) we use the relation for $\gamma=\gamma_{0}(\phi)$ with $\phi$ substituted from $\left(m_{i}, n_{i}\right)$-equations. Also the following relations should be used:

$$
Z_{M N}[* F, g]=-\varepsilon_{g} Z_{M N}[F, g]
$$

(see $(5.10))$ and

$$
G^{i j} d_{j}=\frac{2-d_{0}}{2-D}, \quad G^{i j} l_{j I}=-\sum_{k \in I} \delta_{k}^{i}+\frac{d(I)}{D-2}
$$


where $l_{j I}$ are defined in (3.10). The proposition is proved.

Remark 2. We may also fix the gauge $\gamma=\gamma(\phi)(\gamma(\phi)$ is smooth function) by arbitrary manner or do not fix it. In this case the Proposition 2 is simply modified by the replacement of the action (3.2) by the action (2.32)-(2.34) with $\mathcal{L}_{A}$ from (6.30). This is valid also for $d_{0}=2$ if the restrictions (6.28) and (6.29) are imposed.

\subsection{Exact solutions}

When $\xi_{i}=\Lambda=0$ and all the above constraints are satisfied we deal with $\sigma$-model (3.5), where

$$
S=S_{e} \sqcup S_{m},
$$

$\varepsilon_{s}$ are defined in (3.8), (5.14) and $L_{s}=\left(L_{A s}\right)$ are defined in (3.9) and (5.15) for $s \in S_{e}$ and $s \in S_{m}$ respectively.

Using the Proposition 1 we obtain the exact solutions generalizing pure electric and magnetic ones:

$$
\begin{array}{r}
g=U\left\{g^{0}+\sum_{i=1}^{n} U_{i} g^{i}\right\} \\
U=U_{e} U_{m}, \quad U_{i}=U_{i, e} U_{i, m}, \\
\varphi^{\beta}=\varphi_{e}^{\beta}+\varphi_{m}^{\beta},
\end{array}
$$

where $U_{e}, U_{i, e}, U_{m}, U_{i, m}, \varphi_{e}, \varphi_{m}$ are presented in (3.35), (3.36), (5.17), (5.18), (3.37), (5.19) respectively. The fields of forms are given by (6.1)-(6.4), where

$$
\begin{aligned}
F^{a, e, I}=\nu_{a, e, I} d H_{a, e, I}^{-1} & \wedge \tau(I), \\
F^{b, m, J}=\nu_{b, m, J}\left(*_{0} d H_{b, m, J}\right) & \wedge \tau(\bar{J}),
\end{aligned}
$$

$I \in \Omega_{a, e} ; J \in \Omega_{b, m} ; a \in \Delta_{e} ; b \in \Delta_{m}(\bar{J}$ is defined in (5.25) $)$ and $*_{0} d H$ is the Hodge dual form on $\left(M_{0}, g^{0}\right)$.

Here parameters $\nu_{a, e, I}$ and $\nu_{b, m, J}$ satisfy the relations (3.42), (5.23) respectively. The dimensions (of "branes") $d(I)$ and $\lambda_{a}$ satisfy relations (3.43), (5.24) and the following crossing orthogonality relation

$$
d(I \cap J)+\frac{d(I) d(J)}{2-D}-\lambda_{a} \cdot \lambda_{b}=0
$$

$I \in \Omega_{a, e} ; J \in \Omega_{b, m} ; a \in \Delta_{e} ; b \in \Delta_{m}$ corresponding to 3.15) with $s=(a, e, I) \in S_{e}$, $r=(b, m, J) \in S_{m}$.

All functions $H_{a, e, I}, H_{b, m, J}$ are harmonic on $\left(M_{0}, g^{0}\right)$ :

$$
\Delta\left[g^{0}\right] H_{a, e, I}=0, \quad \Delta\left[g^{0}\right] H_{b, m, J}=0,
$$

$I \in \Omega_{a, e} ; J \in \Omega_{b, m} ; a \in \Delta_{e} ; b \in \Delta_{m}$.

In more compact form relations (3.42), (3.43), (5.23), (5.24), (6.45) read

$$
d\left(I_{s} \cap I_{r}\right)+\frac{d\left(I_{s}\right) d\left(I_{r}\right)}{2-D}+\chi_{s} \chi_{r} \lambda_{a_{s}} \cdot \lambda_{a_{r}}=-\varepsilon_{s} \nu_{s}^{-2} \delta_{s r}
$$


$s, r \in S$. Here we denote $s=\left(a_{s}, o_{s}, I_{s}\right), \nu_{s}=\nu_{a_{s}, o_{s}, I_{s}} ; o_{s}=e, m ; \chi_{s}=+1,-1$ for $s \in S_{e}, S_{m}$ respectively; $I_{s} \in \Omega_{a_{s}, o_{s}}, a_{s} \in \Delta_{o_{s}}, s \in S$.

The solutions (6.40)- (6.47) are valid if the restrictions (4.29), (5.48),

$$
W_{i}^{\left(d_{0}\right)}\left(\Omega_{a, m}, \Omega_{a, e}\right)=\emptyset
$$

$i \in w_{1}$ for $d_{0}=1,3$ (see (6.17), (6.20) ) and (6.28), 6.29) for $d_{0}=2$ are imposed.

\section{$D=12$ model}

Here we illustrate the obtained above general solution by considering a bosonic field model in dimension $D=12$ [49] that admits the bosonic sector of 11-dimensional supergravity as a consistent truncation. The action for this model with omitted Chern-Simons term has the following form

$$
\hat{S}_{12}=\int_{M} d^{12} z \sqrt{|g|}\left\{R[g]-g^{M N} \partial_{M} \varphi \partial_{N} \varphi-\frac{1}{4 !} \exp \left(2 \lambda_{1} \varphi\right)\left(F^{1}\right)^{2}-\frac{1}{5 !} \exp \left(2 \lambda_{2} \varphi\right)\left(F^{2}\right)^{2}\right\} .
$$

Here $\operatorname{rank} F^{1}=4, \operatorname{rankF} F^{2}=5$, and

$$
\lambda_{1}^{2}=-\frac{1}{10}, \quad \lambda_{2}=-2 \lambda_{1}
$$

In (2.1) $\Delta=\{1,2\}$ and all $\theta_{a}=1, a=1,2$. We put $\Delta_{e}=\Delta_{m}=\Delta$, i.e. for both two forms we consider the composite ansatz with electric and magnetic components (see (6.4)).

The dimensions of p-brane worldsheets are

$$
\begin{aligned}
& d(I)=3, \quad I \in \Omega_{1, e}, \\
& \text { 7, } I \in \Omega_{1, m}, \\
& \text { 4, } I \in \Omega_{2, e} \text {, } \\
& \text { 6, } I \in \Omega_{2, m}
\end{aligned}
$$

(see (2.19) and (5.2)). Thus, the model describes electrically charged 2- and 3-branes and magnetically charged 6 - and 5-branes (corresponding to $F^{1}$ and $F^{2}$ respectively).

¿From relations $(6.47)$ we obtain the intersection rules:

$$
\begin{array}{rlrl}
d(I \cap J)=1, & \{d(I), d(J)\}= & \{3,3\},\{3,4\}, \\
2, & & \{3,6\},\{3,7\},\{4,4\},\{4,6\}, \\
3, & \{4,7\}, \\
4, & \{6,6\},\{6,7\}, \\
5, & \{7,7\},
\end{array}
$$

and

$$
\nu_{1, e, I}^{2}=\nu_{1, m, I}^{2}=\nu_{2, e, I}^{2}=\nu_{2, m, I}^{2}=\frac{1}{2}
$$

for all $I$. Also we get

$$
\begin{gathered}
\varepsilon(I)=-1, \quad I \in \Omega_{1, e} \cup \Omega_{2, e}, \\
\varepsilon(J)=\varepsilon_{g}, \quad J \in \Omega_{1, m} \cup \Omega_{2, m},
\end{gathered}
$$


(recall that $\left.\varepsilon_{g} \equiv \operatorname{sign} \operatorname{det}\left(g_{M N}\right)\right)$. Thus, electrically charged $p$-branes should have odd number of time directions and magnetically charged $p$-branes should have even number of time directions for $\varepsilon_{g}=1$ and odd number of time directions for $\varepsilon_{g}=-1$. Relations (6.52) are intersection rules for $p$-branes and relations (6.54), (6.55) are signature restrictions on them. We note that due to relations (6.52) all constraints treated in previous sections are satisfied identically.

The metric (6.40) reads

$$
\begin{array}{r}
g=U_{e} U_{m}\left\{g^{0}+\sum_{i=1}^{n} U_{i, e} U_{i, m} g^{i}\right\} \\
U_{e}=\left(\prod_{I_{1} \in \Omega_{1, e}} H_{1, e, I_{1}}\right)^{\frac{3}{10}}\left(\prod_{I_{2} \in \Omega_{2, e}} H_{2, e, I_{2}}\right)^{\frac{2}{5}}, \\
U_{m}=\left(\prod_{J_{1} \in \Omega_{1, e}} H_{1, m, J_{1}}\right)^{\frac{7}{10}}\left(\prod_{J_{2} \in \Omega_{2, m}} H_{2, m, J_{2}}\right)^{\frac{3}{5}}, \\
U_{i, e}=\left(\prod_{I_{1} \in \Omega_{1, e}, I_{1} \ni i} H_{1, e, I_{1}}^{-1}\right) \prod_{I_{2} \in \Omega_{2, e}, I_{2} \ni i} H_{2, e, I_{2}}^{-1}, \\
U_{i, m}=\left(\prod_{J_{1} \in \Omega_{1, m}, J_{1} \ni i} H_{1, m, J_{1}}^{-1}\right)_{J_{2} \in \Omega_{2, m}, J_{2} \ni i} H_{2, m, J_{2}}^{-1} .
\end{array}
$$

The scalar field is

$$
\varphi=\begin{gathered}
\sum_{I_{1} \in \Omega_{1, e}} \eta \ln H_{1, e, I_{1}}-2 \sum_{I_{2} \in \Omega_{2, e}} \eta \ln H_{2, e, I_{2}} \\
-\sum_{J_{1} \in \Omega_{1, m}} \eta \ln H_{1, m, J_{1}}+2 \sum_{J_{2} \in \Omega_{2, m}} \eta \ln H_{2, m, J_{2}},
\end{gathered}
$$

where $\eta=\lambda_{1} / 2$. The fields of forms are the following

$$
\begin{aligned}
F^{1} & =\sum_{I_{1} \in \Omega_{1, e}} \nu_{1, e, I_{1}} d H_{1, e, I_{1}}^{-1} \wedge \tau\left(I_{1}\right)+\sum_{J_{1} \in \Omega_{1, m}} \nu_{1, m, J_{1}}\left(*_{0} d H_{1, m, J_{1}}\right) \wedge \tau\left(\bar{J}_{1}\right), \\
F^{2} & =\sum_{I_{2} \in \Omega_{2, e}} \nu_{2, e, I_{2}} d H_{2, e, I_{2}}^{-1} \wedge \tau\left(I_{2}\right)+\sum_{J_{2} \in \Omega_{2, m}} \nu_{2, m, J_{2}}\left(*_{0} d H_{2, m, J_{2}}\right) \wedge \tau\left(\bar{J}_{2}\right),
\end{aligned}
$$

where $*_{0} d H$ is the Hodge dual form on $\left(M_{0}, g^{0}\right)$. The metric and all fields are defined on manifold (2.10) and all functions $H_{a, o, I}$ are harmonic on $M_{0}$.

The subsets $\Omega_{a, o} \in \Omega$ satisfy the intersection rules (6.52) and the signature restrictions (6.54), (6.55).

These solutions also satisfy the equations of motion for $D=12$ model from ref. [49] with Chern-Simons term included. This can be readily verified using the relation for the total action

$$
S_{12}=\hat{S}_{12}+c_{12} \int_{M} A^{2} \wedge F^{1} \wedge F^{1}
$$

where $c_{12}=$ const and $\hat{S}_{12}$ is defined in 6.49$)\left(F^{2}=d A^{2}\right)$. Note that the elementary p-brane solutions corresponding to $F^{1}$ field $(p=2,6)$ were considered in 49. 


\section{$D=11$ supergravity}

Now we consider as another example the $D=11$ supergravity [1, 2]. The action for the bosonic sector of this theory with omitted Chern-Simons term has the following form

$$
\hat{S}_{11}=\int_{M} d^{11} z \sqrt{|g|}\left\{R[g]-\frac{1}{4 !}(F)^{2}\right\} .
$$

Here $\operatorname{rank} F=4$.

The dimensions of p-brane worldsheets are (see (2.19) and (5.2))

$$
\begin{aligned}
d(I)=\quad 3, \quad I \in \Omega_{e}, \\
6, \quad I \in \Omega_{m} .
\end{aligned}
$$

The model describes electrically charged 2-branes and magnetically charged 5-branes.

¿From (6.47) we obtain the intersection rules [18]

$$
\begin{aligned}
& d(I \cap J)=1,\{d(I), d(J)\}=\{3,3\}, \\
& 2, \quad\{3,6\} \text {, } \\
& 4, \quad\{6,6\} \text {, }
\end{aligned}
$$

and

$$
\nu_{e, I}^{2}=\nu_{m, I}^{2}=\frac{1}{2}
$$

for all $I$. Here and below we omitted the index "1" numerating forms. Also we get

$$
\begin{aligned}
& \varepsilon(I)=-1, \quad I \in \Omega_{e}, \\
& \varepsilon(J)=\varepsilon_{g}, \quad J \in \Omega_{m} .
\end{aligned}
$$

The stress-tensor restrictions are also satisfied for these solutions.

The metric (6.49) reads

$$
\begin{array}{r}
g=U_{e} U_{m}\left\{g^{0}+\sum_{i=1}^{n} U_{i, e} U_{i, m} g^{i}\right\}, \\
U_{e}=\left(\prod_{I \in \Omega_{e}} H_{e, I}\right)^{\frac{1}{3}}, \quad U_{m}=\left(\prod_{J \in \Omega_{e}} H_{m, J}\right)^{\frac{2}{3}}, \\
U_{i, e}=\prod_{I \in \Omega_{e}, I \ni i} H_{e, I}^{-1}, \quad U_{i, m}=\prod_{J \in \Omega_{m}, J \ni i} H_{m, J}^{-1} .
\end{array}
$$

The fields of forms are following

$$
F=\sum_{I \in \Omega_{e}} \nu_{e, I} d H_{e, I}^{-1} \wedge \tau(I)+\sum_{J \in \Omega_{m}} \nu_{m, J}\left(*_{0} d H_{m, J}\right) \wedge \tau(\bar{J}),
$$

where $*_{0} d H$ is the Hodge dual form on $\left(M_{0}, g^{0}\right)$. The metric and all fields are defined on manifold (2.10) and all functions $H_{o, I}, o=e, m$, are harmonic on $M_{0}$.

The subsets $\Omega_{o} \in \Omega, o=e, m$, satisfy the intersection rules (6.67) and the signature restrictions (6.69), (6.70). 
The solutions also satisfy the equations of motion with Chern-Simon term taken into account. This can be readily verified using the relation for the bosonic part of action for $D=11$ supergravity [1, 2]

$$
S_{11}=\hat{S}_{11}+c_{11} \int_{M} A \wedge F \wedge F
$$

where $c_{11}=$ const and $\hat{S}_{11}$ is defined in $(6.65)(F=d A)$. We note that these solutions (see also [26]) coincide with those obtained in [27, 18]) for flat $M_{\nu}, \nu=0, \ldots, n$.

\section{Generalization to non-Ricci-flat spaces}

Here we present a generalization of the above solution to the case of non-Ricci flat space $\left(M_{0}, g^{0}\right)$ and when some additional internal Einstein spaces of non-zero curvature $\left(M_{i}, g^{i}\right)$, $i=n+1, \ldots, n+k$, are included.

\subsection{Non-Ricci-flat solutions for $\sigma$-model with a potential}

Let us consider the $\sigma$-model governed by the action

$$
\begin{aligned}
S_{\sigma}=S_{\sigma}\left[g^{0}, \sigma, \Phi\right]=\int_{M_{0}} d^{d_{0}} x \sqrt{\left|g^{0}\right|}\left\{R\left[g^{0}\right]\right. & -\hat{G}_{A B} g^{0 \mu \nu} \partial_{\mu} \sigma^{A} \partial_{\nu} \sigma^{B}-2 V(\sigma) \\
& \left.-\sum_{s \in S} \varepsilon_{s} \mathrm{e}^{2 L_{A s} \sigma^{A}} g^{0 \mu \nu} \partial_{\mu} \Phi^{s} \partial_{\nu} \Phi^{s}\right\} .
\end{aligned}
$$

Here $\left(\hat{G}_{A B}\right)$ is non-degenerate matrix and $\varepsilon_{s} \neq 0, s \in S(S \neq \emptyset)$.

The equations of motion for the action (7.1) have the following form

$$
\begin{array}{r}
R_{\mu \nu}\left[g^{0}\right]=\hat{G}_{A B} \partial_{\mu} \sigma^{A} \partial_{\nu} \sigma^{B}+\frac{2 V}{d_{0}-2} g_{\mu \nu}^{0}+\sum_{s \in S} \varepsilon_{s} \mathrm{e}^{2 L_{A s} \sigma^{A}} \partial_{\mu} \Phi^{s} \partial_{\nu} \Phi^{s} \\
\hat{G}_{A B} \Delta\left[g^{0}\right] \sigma^{B}-\frac{\partial V}{\partial \sigma^{A}}-\sum_{s \in S} \varepsilon_{s} L_{A s} \mathrm{e}^{2 L_{C s} \sigma^{C}}\left(\partial \Phi^{s}\right)^{2}=0 \\
\partial_{\mu}\left(\sqrt{\left|g^{0}\right|} g^{0 \mu \nu} \mathrm{e}^{2 L_{A s} \sigma^{A}} \partial_{\nu} \Phi^{s}\right)=0
\end{array}
$$

$s \in S$. In what follows we consider the potential of a special form

$$
V=V(\sigma)=\sum_{c=1}^{k} A_{c} \exp \left(u_{c A} \sigma^{A}\right)
$$

where $A_{c} \neq 0$ and vectors $u_{c}=\left(u_{c A}\right)$ satisfy the orthogonality conditions

$$
\hat{G}^{A B} u_{c A} L_{B s}=0
$$

$c=1, \ldots, k ; s \in S$.

We also consider the "truncated" action

$$
S_{\sigma, 0}=S_{\sigma, 0}\left[g^{0}, \hat{\sigma}\right]=\int_{M_{0}} d^{d_{0}} x \sqrt{\left|g^{0}\right|}\left\{R\left[g^{0}\right]-\hat{G}_{A B} g^{0 \mu \nu} \partial_{\mu} \hat{\sigma}^{A} \partial_{\nu} \hat{\sigma}^{B}-2 V(\hat{\sigma})\right\},
$$


i.e. the action (7.1) with $\Phi^{s}=0, s \in S$, and the action (7.1) with omitted curvature and potential terms

$$
S_{\sigma, 1}=S_{\sigma, 1}\left[g^{0}, \bar{\sigma}, \Phi\right]=\sqrt{\left|g^{0}\right|}\left\{-\hat{G}_{A B} g^{0 \mu \nu} \partial_{\mu} \bar{\sigma}^{A} \partial_{\nu} \bar{\sigma}^{B}-\sum_{s \in S} \varepsilon_{s} \mathrm{e}^{2 L_{A s} \bar{\sigma}^{A}} g^{0 \mu \nu} \partial_{\mu} \Phi^{s} \partial_{\nu} \Phi^{s}\right\}
$$

Proposition 3. Let us consider the action (7.1), with the potential (7.5) satisfying orthogonality relations (7.6). Let metric $g^{0}$ and $\hat{\sigma}=\left(\hat{\sigma}^{A}(x)\right)$ satisfy equations of motion for the action (7.7) and the constraints imposed:

$$
L_{A s} \hat{\sigma}^{A}=0, \quad s \in S .
$$

Let $g^{0}, \bar{\sigma}=\left(\bar{\sigma}^{A}(x)\right)$ and $\Phi=\left(\Phi^{s}(x)\right)$ satisfy the equations of motion for the action (7.8) and

$$
\bar{\sigma}^{A}=L_{s}^{A} f^{s}
$$

where $f^{s}=f^{s}(x)$ are some functions, $L_{s}^{A}=\hat{G}^{A B} L_{B s},\left(\hat{G}^{A B}\right)=\left(\hat{G}_{A B}\right)^{-1}, s \in S$. Then, the field configuration

$$
g^{0}, \quad \sigma=\hat{\sigma}+\bar{\sigma}, \quad \Phi
$$

satisfies the equations of motion (7.2)-(7.4).

Proof. The proposition can be readily verified using the relations

$$
\begin{array}{r}
\hat{G}_{A B} \partial_{\mu} \bar{\sigma}^{A} \partial_{\nu} \hat{\sigma}^{B}=0 \\
V(\hat{\sigma}+\bar{\sigma})=V(\hat{\sigma}), \quad \frac{\partial}{\partial \sigma^{A}} V(\hat{\sigma}+\bar{\sigma})=\frac{\partial}{\partial \sigma^{A}} V(\hat{\sigma}) ; \\
L_{A s}\left(\bar{\sigma}^{A}+\hat{\sigma}^{A}\right)=L_{A s} \bar{\sigma}^{A}
\end{array}
$$

following from the conditions of Proposition 3.

Thus, we may find the exact solutions by two steps. First, we should solve the equations of motion for the "truncated" model (7.7) and find "background" $\left(\hat{\sigma}, g^{0}\right)$ satisfying (7.9). On the second stage we should solve the equations of motions corresponding to (7.8) for the fields $\bar{\sigma}$ and $\Phi$ on $\left(M_{0}, g^{0}\right)$-background with the restriction of vanishing of total energy-momentum tensor for $(\bar{\sigma}, \Phi)$-fields.

\subsection{Generalized intersecting $p$-brane solutions with non-Ricci- flat spaces}

Here we apply the scheme considered in Subsect. 7.1 to the model (2.1) with $\Lambda=0$. Now the manifold is

$$
M=M_{0} \times M_{1} \times \ldots \times M_{n} \times M_{n+1} \times \ldots \times M_{n+k}
$$

instead of (2.10) and the metric

$$
g=\mathrm{e}^{2 \gamma(x)} g^{0}+\sum_{i=1}^{n+k} \mathrm{e}^{2 \phi^{i}(x)} g^{i}
$$

instead of (2.11). 
All $\left(M_{i}, g^{i}\right)$ are Einstein spaces, satisfying (2.12), $i=1, \ldots, n+k$, with

$$
\xi_{1}=\ldots=\xi_{n}=0, \quad \xi_{n+1} \neq 0, \ldots, \xi_{n+k} \neq 0 .
$$

Then for electro-magnetic $p$-brane ansatz from Section 6 we get according to Proposition 2 the $\sigma$-model (7.1) with midisupermetric (3.6); $S, \varepsilon_{s}$ and $L_{A s}$ are defined in (6.39), (3.8), (5.14) and (3.9), (5.15) respectively and $i, j=1, \ldots, n+k$.

The potential $V(\sigma), \sigma=\left(\phi^{i}, \varphi^{\alpha}\right)$, in this case has the form (7.5) with

$$
\begin{array}{r}
u_{c i}=-2 \delta_{i}^{n+c}+\frac{2 d_{i}}{2-d_{0}}, \quad u_{c \alpha}=0, \\
A_{c}=-\frac{1}{2} \xi_{n+c} d_{n+c},
\end{array}
$$

$c=1, \ldots, k ; i=1, \ldots, n+k ; \alpha=1, \ldots, l$.

It may be verified that the vectors $u_{c}=\left(u_{c A}\right)$ from (7.18) satisfy the orthogonality condition (7.6). Indeed, the calculation gives for $s=(a, o, I)\left(o=e, m\right.$ and $\left.I \in \Omega_{a, o}\right)$

$$
\hat{G}^{A B} u_{c A} L_{B s}=2 \frac{d(\{n+c\} \cap I)}{d_{n+c}}=0,
$$

since $\{n+c\} \cap I=\emptyset$ for $c=1, \ldots, k$ and $I \in\{1, \ldots, n\}$.

Here $\Omega=\Omega(n)$ is unchanged, so all $p$-branes do not "live" in non-Ricci-flat "internal" spaces $\left(M_{n+c}, g^{n+c}\right), c=1, \ldots, k$.

Then from Propositions 1, 3 and the results of Section 6 we obtain new exact solutions with the metric

$$
g=U\left\{\mathrm{e}^{2 \hat{\gamma}(x)} g^{0}+\sum_{i=1}^{n+k} U_{i} \mathrm{e}^{2 \hat{\phi}^{i}(x)} g^{i}\right\}
$$

instead of $(6.40)$ and scalar field

$$
\varphi^{\beta}=\hat{\varphi}^{\beta}+\varphi_{e}^{\beta}+\varphi_{m}^{\beta}
$$

instead of (6.42). In (7.21) $U, U_{i}, i=1, \ldots, n$, are defined in 6.41) (here $D=\sum_{i=0}^{n+k} d_{i}$ ), $U_{n+1}=\ldots=U_{n+k}=1$, and

$$
\hat{\gamma}=\gamma_{0}(\hat{\phi})=\frac{1}{2-d_{0}} \sum_{i=1}^{n+k} d_{i} \hat{\phi}^{i} .
$$

The background fields $g^{0}$ and $\left(\hat{\sigma}^{A}\right)=\left(\hat{\phi}^{i}(x), \hat{\varphi}^{\alpha}(x)\right)$ satisfy the equations of motion for the $\sigma$-model (7.7) with $\left(G_{A B}\right)$ defined in (3.6) and $\left(G_{i j}\right)$ in $(3.3), i, j=1, \ldots, n+k$, and

$$
V(\hat{\sigma})=-\frac{1}{2} \sum_{i=n+1}^{n+k} \xi_{i} d_{i} e^{-2 \hat{\phi}^{i}+2 \gamma_{0}(\hat{\phi})} .
$$

In other words the metric

$$
\hat{g}=\mathrm{e}^{2 \hat{\gamma}(x)} g^{0}+\sum_{i=1}^{n+k} \mathrm{e}^{2 \hat{\phi}^{i}(x)} g^{i}
$$


and the set of scalar fields $\hat{\varphi}=\left(\hat{\varphi}^{\beta}(x)\right)$ should satisfy the equations of motion for the action (2.1) with $\Lambda=0$ and $F^{a}=0, a \in \Delta$. Background fields should also satisfy the constraints (following from $(7.9)$ )

$$
\begin{aligned}
\lambda_{a}(\hat{\varphi})-\sum_{i \in I} d_{i} \hat{\phi}^{i}=0, & I \in \Omega_{a, e}, \quad a \in \Delta_{e}, \\
-\lambda_{b}(\hat{\varphi})-\sum_{j \in J} d_{j} \hat{\phi}^{j}=0, \quad J \in \Omega_{b, m}, & b \in \Delta_{m} .
\end{aligned}
$$

Relations (7.21), (7.22), (7.26), (7.27) are the only modifications of the solutions from Section 6. (All other relations for $F^{a}, \nu_{s}, \ldots$ are unchanged).

\section{Concluding remarks}

Using $\sigma$-model approach we have obtained generalized composite electro-magnetic p-brane solutions. The solutions (3.34) with flat spaces $\left(M_{\nu}, g^{\nu}\right)$ one of which being pseudoEuclidean one: $M_{\nu}=\mathbf{R}^{d_{\nu}}, g^{0}=\delta_{\mu \nu} d x^{\mu} \otimes d x^{\nu}, g^{1}=\eta_{m_{1} n_{1}} d y_{1}^{m_{1}} \otimes d y_{1}^{n_{1}}, g^{i}=\delta_{m_{i} n_{i}} d y_{i}^{m_{i}} \otimes d y_{i}^{n_{i}}$ $(i>1)$ and

$$
H_{s}(x)=1+\sum_{k=1}^{N_{s}} \frac{2 m_{s k}}{\left|x-x_{s k}\right|^{d_{0}-2}}, \quad s \in S,
$$

are usually interpreted in literature as intersecting p-branes when all sets $I$ contain 1 . In this case all p-branes have common intersection containing $M_{1}$ manifold and time submanifold belongs to worldsheets of all p-branes.

Our solution may be considered as a generalization of intersecting p-brane solutions to the case of Ricci-flat (and also for some non-Ricci-flat) manifolds $\left(M_{\nu}, g^{\nu}\right)$ of arbitrary signatures. In this case the submanifolds $M_{I}$ (2.16) may be not intersecting and may contain different time submanifolds, i.e. p-branes may "live" in different times. They may be considered as a starting point for a generalization of multitemporal sphericallysymmetric solutions 46, 47] to the standard p-brane case.

\section{Acknowledgments}

This work was supported in part by DFG grants 436 RUS 113/7, 436 RUS 113/236/O(R) and by the Russian Ministry for Science and Technology, Russian Fund for Basic Research, project N 95-02-05785-a. The authors are grateful to Dr. M.Rainer for his hospitality during their stay in Potsdam University and to K.A.Bronnikov for valuable comments.

\section{References}

[1] E. Cremmer, B.Julia, and J. Scherk, Phys. Lett. B76, 409 (1978).

[2] A. Salam and E. Sezgin, eds., "Supergravities in Diverse Dimensions", reprints in 2 vols., World Scientific (1989).

[3] M.B. Green, J.H. Schwarz, and E. Witten, "Superstring Theory" in 2 vols. (Cambridge Univ. Press, 1987). 
[4] C. Hull and P. Townsend, "Unity of Superstring Dualities", Nucl. Phys. B 438, 109 (1995), Preprint hep-th/9410167;

P.K. Townsend, Phys. Lett. B 350, 184 (1995), Preprint hep-th/9501068;

E. Witten, Nucl. Phys. B 443, 85 (1995), Preprint hep-th/9503124;

P. Horava and E. Witten, Nucl. Phys. B 460, 506 (1996), Preprint hep-th/9510209; Preprint hep-th/9603142.

[5] J.M. Schwarz, "Lectures on Superstring and M-theory Dualities", Preprint ICTP, hepth/9607201.

[6] M.J. Duff, "M-theory (the Theory Formerly Known as Strings)", Preprint CTP-TAMU$33 / 96$, hep-th/9608117.

[7] C.M. Hull, "String dynamics at strong coupling" Nucl. Phys. B 468, 113 (1996);

C. Vafa, "Evidence for F-Theory", Preprint hep-th/9602022; Nucl. Phys. B 469, 403 (1996).

[8] A. Dabholkar, G. Gibbons, J.A. Harvey, and F. Ruiz Ruiz, Nucl. Phys. B 340, 33 (1990).

[9] C.G. Callan, J.A. Harvey and A. Strominger, Nucl. Phys. 359 (1991) 611; Nucl. Phys. B 367, 60 (1991).

[10] M.J. Duff and K.S. Stelle, Phys. Lett. B 253, 113 (1991).

[11] G.T. Horowitz and A. Strominger, Nucl. Phys. B 360, 197 (1991).

[12] R. Güven, Phys. Lett. B 276, 49 (1992); Phys. Lett. B 212, 277 (1988).

[13] H. Lü, C.N. Pope, E. Sezgin and K. Stelle, Nucl. Phys. B 456, 669 (1995).

[14] M.J. Duff, R.R. Khuri and J.X. Lu, Phys. Rep. 259, 213 (1995).

[15] G.W. Gibbons, G.T. Horowitz and P.K. Townsend, Class. Quant. Grav. 12, 297 (1995); Preprint hep-th/9410073

[16] A.A. Tseytlin, Mod. Phys. Lett. A11, 689 (1996); Preprint hep-th/9601177.

[17] G. Papadopoulos and P.K. Townsend, "Intersecting M-branes", Preprint hep-th/9603087; Phys. Lett. B 380, 273 (1996).

[18] A.A. Tseytlin, "Harmonic Superpositions of M-branes", Preprint hep-th/9604035; Nucl. Phys. B 475 (1996) 149.

[19] J.P. Gauntlett, D.A. Kastor, and J. Traschen, "Overlapping Branes in M-Theory", Preprint hep-th/9604179; Nucl. Phys. B 478, 544 (1996).

[20] I.R. Klebanov and A.A. Tseytlin, "Intersecting $M$-branes as Four-Dimensional Black Holes", Preprint PUPT-1616, Imperial/TP/95-96/41, hep-th/9604166; Nucl. Phys. B 475, 164 (1996).

[21] H. Lü, C.N. Pope, and K.S.Stelle, "Vertical Versus Diagonal Reduction for p-Branes", Preprint hep-th/9605082.

[22] N. Khvengia, Z. Khvengia, H. Lü, C.N. Pope, "Intersecting M-Branes and Bound States", Preprint hep-th/9605082. 
[23] E. Bergshoeff, R. Kallosh and T. Ortin, "Stationary Axion/Dilaton Solutions and Supersymmetry", Preprint hep-th/9605059; Nucl. Phys. B 478, 156 (1996).

[24] G. Clément and D.V. Gal'tsov, "Stationary BPS solutions to dilaton-axion gravity" Preprint GCR-96/07/02 DTP-MSU/96-11, hep-th/9607043.

[25] I. Aref'eva and A. Volovich, "Composite p-branes in Diverse Dimensions", Preprint SMI19-96, hep-th/9611026.

[26] V.D.Ivashchuk and V.N.Melnikov, "Intersecting p-brane Solutions in Multidimensional Gravity and M-theory", Preprint hep-th/9612089; Gravitation and Cosmology 2, No 4, 204 (1996);

V.D.Ivashchuk and V.N.Melnikov, Phys. Lett. B (1997) to be published.

[27] E. Bergshoeff, M. de Roo, E. Eyras, B. Janssen and J.P. van der Schaar, Preprint hepth/9612095.

[28] I.Ya. Aref'eva and O.A. Rytchkov, "Incidence Matrix Description of Intersecting p-brane Solutions", Preprint SMI-25-96, hep-th/9612236.

[29] R. Argurio, F. Englert and L. Hourant, "Intersection Rules for p-branes", hep-th/9701042.

[30] K.S. Stelle, "Lectures on Supergravity p-branes ", hep-th/9701088.

[31] I.Ya. Aref'eva, K. Viswanathan, A.I. Volovich and I.V. Volovich, "p-Brane Solutions in Diverse Dimensions", Preprint hep-th/9701092.

[32] I.Ya. Aref'eva, M.G. Ivanov and O.A. Rytchkov, "Properties of Intersecting p-branes in Various Dimensions", Preprint SMI-05-97, hep-th/9702077.

[33] V.D. Ivashchuk, V.N. Melnikov and M. Rainer, "Multidimensional $\sigma$-models with Composite Electric p-branes", gr-qc/9705005.

[34] S.D. Majumdar, Phys. Rev. 72, 930 (1947);

A. Papapetrou, Proc. R. Irish Acad. A51, 191 (1947).

[35] N.M.Bocharova, K.A. Bronnikov and V.N. Melnikov, Vestnik MGU (Moscow Univ.), 6, 706 (1970)(in Russian) - first MP-type solution with conformal scalar field;

K.A. Bronnikov, Acta Phys. Polonica , B4, 251 (1973);

K.A. Bronnikov and V.N. Melnikov, in Problems of Theory of Gravitation and Elementary Particles , 5, 80 (1974) (in Russian) - first MP-type solution with conformal scalar and electromagnetic fields;

K.A. Bronnikov, V.N. Melnikov, G.N. Shikin and K.P.Stanyukovich, Ann. Phys., 118, 84 (1979) - first MP-type solution with dilatonic type interaction of scalar and electromagnetic fields;

K.A. Bronnikov, V.N. Melnikov, Ann. Phys., 239, 40 (1995) - multidimensional MP-type solutions and stability analysis.

[36] V.A. Berezin, G. Domenech, M.L. Levinas, C.O. Lousto and N.D. Umerez, Gen. Relativ. Grav. 21, 1177 (1989).

[37] M. Szydłowski, Acta Cosmologica 18, 85 (1992). 
[38] M. Rainer and A. Zhuk, Phys. Rev. D, 546186 (1996).

[39] V.D. Ivashchuk and V.N. Melnikov, "Multidimensional Gravity with Einstein Internal spaces", preprint RGS-96-003; Gravitation and Cosmology 2 No 3 (7), 177 (1996).

[40] K.A. Bronnikov and J.C. Fabris, Gravitation and Cosmology 2, No 4 (8), (1996).

[41] V.D. Ivashchuk, V.N. Melnikov, Phys. Lett. A135, 465 (1989).

[42] V.D. Ivashchuk, V.N. Melnikov and A.I. Zhuk, Nuovo Cimento B104, 575 (1989).

[43] V.D. Ivashchuk and V.N. Melnikov, "Extremal Dilatonic Black Holes in String-like Model with Cosmological Term", Preprint RGS-96-002; hep-th/9603107; Phys. Lett. B 384, 58 (1996).

[44] G.W. Gibbons and S.W. Hawking, Phys. Rev. D 15, 2752 (1977).

[45] V.D. Ivashchuk and V.N. Melnikov, Int. J. Mod. Phys. D 3, No 4, 795 (1994);

V.D. Ivashchuk and V.N. Melnikov, Gravitation and Cosmology 1, No 3, 204 (1995);

V.R. Gavrilov, V.D. Ivashchuk and V.N. Melnikov, J. Math. Phys 36, 5829 (1995);

V.N. Melnikov, "Multidimensional Cosmology and Gravitation", Preprint CBPF, Rio de Janeiro, 1995, 210p.

[46] V.D. Ivashchuk and V.N. Melnikov, Class. and Quant. Grav. 11, 1793 (1994).

[47] V.D. Ivashchuk and V.N. Melnikov, Int. J. Mod. Phys. D 4, 167 (1995).

[48] S. Hewson and M. Perry, "The Twelve-Dimensional Super (2+2)-Brane ", Preprint hepth/9612008.

[49] N. Khvengia, Z. Khvengia and C.N. Pope, "Toward Field Theory of F-Theory" Preprint hep-th/9703012.

[50] H. Nishino, "Supergravity in 10+2 Dimensions as Consistent Background for Superstring", Preprint UMDEPP 97-101, hep-th/9703214. 